\title{
A Jacobi Collocation Method for the Fractional Ginzburg-Landau Differential Equation
}

\author{
Yin Yang ${ }^{1, *}$, Jianyong Tao ${ }^{1}$, Shangyou Zhang ${ }^{2}$ and Petr V. Sivtsev ${ }^{3}$ \\ ${ }^{1}$ Hunan Key Laboratory for Computation and Simulation in Science and Engineering, \\ Key Laboratory of Intelligent Computing $\mathcal{E}$ Information Processing of Ministry of \\ Education, School of Mathematics and Computational Science, Xiangtan University, \\ Xiangtan, Hunan 411105, China \\ 2 Department of Mathematical Sciences, University of Delaware, Newark, DE 19716, \\ USA \\ ${ }^{3}$ International Scientific and Research Laboratory of Multiscale Model Reduction and \\ High Performance Computing, Ammosov North Eastern University, Kulakovskogo, 677013, \\ Yakutsk, Russia
}

Received 14 March 2019; Accepted (in revised version) 7 June 2019

\begin{abstract}
In this paper, we design a collocation method to solve the fractional Ginzburg-Landau equation. A Jacobi collocation method is developed and implemented in two steps. First, we space-discretize the equation by the Jacobi-GaussLobatto collocation (JGLC) method in one- and two-dimensional space. The equation is then converted to a system of ordinary differential equations (ODEs) with the time variable based on JGLC. The second step applies the Jacobi-Gauss-Radau collocation (JGRC) method for the time discretization. Finally, we give a theoretical proof of convergence of this Jacobi collocation method and some numerical results showing the proposed scheme is an effective and high-precision algorithm.
\end{abstract}

AMS subject classifications: 35R35, 65M12, 65M70

Key words: The fractional Ginzburg-Landau equation, Jacobi collocation method, convergence.

\section{Introduction}

The fractional Ginzburg-Landau equation (FGLE) is known as a generalization of the classical one and has been presented to depict many kinds of nonlinear phenomena. The Ginzburg-Landau equation (GLE) has a variety of applications, e.g., in biology and chemistry. In many areas of physics, the GLE also has important applications, such as superconductivity, superfluidity, nonlinear optics, Bose-Einstein condensation and so on [1].

*Corresponding author.

Email: yangyinxtu@xtu.edu.cn (Y. Yang) 
At first, Ginzburg and Landau proposed the GLE to depict phase transitions in superconductors near their critical temperature. The GLE also models the dynamics of electromagnetic behavior of a superconductor in an external magnetic field [2].

In recent years, as the fractional differential equations have many applications in different fields of engineering and science, it attracted more and more scholars. A fractional Ginzburg-Landau equation is derived by Tarasov et al. [2] from the variational EulerLagrange equation for fractal media. Because fractals generate in a fractal media or a fractal process in nature, the FGLE has been used to depict many physical phenomena, for example, the dynamical processes in continuum with fractal dispersion or in media with a fractal mass dimension [2], the organization of a system near the phase transition point influenced by a competing nonlocal ordering [4], and a network of diffusively Hindmarsh-Rose neurons with a long-range synaptic coupling [5].

In this paper, we consider a numerical algorithm for solving the following GinzburgLandau equation with fractional Laplace operator $(1<a \leq 2)$ :

$$
u_{t}+(v+i \eta)(-\Delta)^{\frac{a}{2}} u+(k+i \zeta)|u|^{2} u-\gamma u=0, \quad x \in \mathbb{R}, \quad t \in[0, T]
$$

and the initial condition

$$
u(x, 0)=u_{0}(x), \quad x \in \mathbb{R},
$$

where $i^{2}=-1, u(x, t)$ is the unknown complex function from $\mathbb{R} \times \mathbb{R}^{+}$to $\mathbb{C}, u_{0}(x)$ is a given smooth function, and $v>0, k>0, \eta, \zeta, \gamma$ are real constants. The fractional Laplace operator $(-\Delta)^{\frac{a}{2}}$ can be defined with the symbol $|\xi|^{a}$ as follows:

$$
-(-\Delta)^{\frac{a}{2}} u(x, t)=-\mathcal{F}^{-1}\left(|\xi|^{a} \tilde{u}(\xi, t)\right),
$$

where $\tilde{u}$ is the Fourier transform of $u$ and $\mathcal{F}$ denotes the Fourier transform operator. Yang et al. $[6,7]$ showed that the fractional derivative defined in Eq. (1.3) is equivalent to the Riesz fractional derivative, i.e.,

$$
\begin{aligned}
& \frac{\partial^{a}}{\partial|x|^{a}} u(x, t)=-(-\Delta)^{\frac{a}{2}} u(x, t) \\
= & -\frac{1}{2 \cos \left(\frac{a \pi}{2}\right)}\left[-\infty{ }^{R} D_{x}^{a} u(x, t)+{ }_{x}^{R} D_{+\infty}^{a} u(x, t)\right] .
\end{aligned}
$$

The left Riemann-Liouville fractional derivative of $u(x, t)$ is defined as follows:

$$
{ }_{-\infty}^{R} D_{x}^{a} u(x, t)= \begin{cases}\frac{1}{\Gamma(2-a)} \frac{\partial^{2}}{\partial x^{2}} \int_{-\infty}^{x}(x-s)^{1-a} u(s, t) d s, & 1<a<2, \\ \frac{\partial^{2}}{\partial x^{2}} u(x, t), & a=2,\end{cases}
$$


and the right Riemann-Liouville fractional derivative of $u(x, t)$ as:

$$
{ }_{x}^{R} D_{+\infty}^{a} u(x, t)= \begin{cases}\frac{1}{\Gamma(2-a)} \frac{\partial^{2}}{\partial x^{2}} \int_{x}^{+\infty}(s-x)^{1-a} u(s, t) d s, & 1<a<2, \\ \frac{\partial^{2}}{\partial x^{2}} u(x, t), & a=2 .\end{cases}
$$

Some theoretical analyses for the FGLE (1.1) are given in [10-13]. The global wellposedness was investigated by $\mathrm{Pu}$ and Guo [14]; The psi-series solution was derived and analyzed by Tarasov [13]. Guo and Huo [11] studied the inviscid limit behavior of (1.1) to the fractional Schrödinger equation. The well-posedness and asymptotic behaviors in two-dimensions are given in [10].

We know the spectral collocation method is one of the high-precision numerical approaches for solving the fractional differential equations (FDEs) [9]. We develop collocation methods for solving one-dimensional (1D) and two-dimensional (2D) FGLE. Firstly, we proposed JGLC method to discrete the FGLE in space so that the FGLE with an initial condition is converted to a system of ODEs. Secondly, we deal with the system of ODEs by a JGRC method. We will give a convergence analysis of the collocation method. Finally, several numerical examples in 1D and 2D are presented, verifying the theoretical analysis. They indicate the high precision and effectiveness of the algorithm.

The main layout of this article as follows: we mainly introduce the basic properties and recursive relations of Jacobi polynomials in the Section 2. We define the JGLC method to solve the 1D and 2D fractional Ginzburg-Landau equation in Section 3. We use the JGRC method to deal with the ordinary differential equations with initial conditions in Section 4. Next, the convergence analysis of the collocation method is investigated in Section 5. In Section 6, we give several numerical examples for testing precision of the method for the fractional Ginzburg-Landau equation in 1D and 2D. Finally, some concluding remarks are drawn in Section 7.

\section{Some properties of Jacobi polynomials}

In this section, we will introduce some basic properties about Jacobi polynomials that are most related to spectral collocation methods [16].

The Jacobi polynomials, denoted by $\mathscr{P}_{n}^{(\alpha, \beta)}(x)$, are orthogonal with the Jacobi weight function $\omega^{(\alpha, \beta)}(x)=(1-x)^{\alpha}(1+x)^{\beta}$ over $I=(-1,1)$, namely,

$$
\left(\mathscr{P}_{i}^{(\alpha, \beta)}(x), \mathscr{P}_{j}^{(\alpha, \beta)}(x)\right)_{\omega^{(\alpha, \beta)}}=\int_{-1}^{1} \mathscr{P}_{i}^{(\alpha, \beta)}(x) \mathscr{P}_{j}^{(\alpha, \beta)}(x) \omega^{(\alpha, \beta)}(x) d x=h_{i}^{(\alpha, \beta)} \delta_{i j}
$$

where $\delta_{i, j}$ is the Kronecker function and

$$
h_{i}^{(\alpha, \beta)}=\frac{(2)^{\alpha+\beta+1} \Gamma(i+\alpha+1) \Gamma(i+\beta+1)}{(2 i+\alpha+\beta+1) \Gamma(i+1) \Gamma(i+\alpha+\beta+1)} .
$$


The expression of the Jacobi polynomial is as follows:

$$
\mathscr{P}_{n}^{(\alpha, \beta)}(x)=\frac{(-1)^{n}}{2^{n} n !}(1-x)^{-\alpha}(1+x)^{-\beta} \frac{d^{n}}{d x^{n}}\left[(1-x)^{\alpha+n}(1+x)^{\beta+n}\right], \quad n \in N^{+}, \quad \alpha, \beta>-1 .
$$

We show that the Jacobi polynomials are the eigenfunctions of the singular SturmLiouville problem:

$$
\left(x^{2}-1\right) \partial_{x}^{2} u(x)+(\alpha-\beta-(\alpha+\beta+2) x) \partial_{x} u(x)=\lambda_{n}^{\alpha, \beta} \mathscr{P}_{n}^{(\alpha, \beta)}(x), \quad x \in[-1,1],
$$

and the corresponding eigenvalue is:

$$
\lambda_{n}^{\alpha, \beta}=n(n+\alpha+\beta+1) .
$$

The Jacobi polynomials $\mathscr{P}_{i}^{(\alpha, \beta)},(i=0,1, \cdots)$ with the parameters $(\alpha>-1, \beta>-1)$ satisfy the following relations:

$$
\begin{aligned}
& \mathscr{P}_{i+1}^{(\alpha, \beta)}(x)=\left(a_{i}^{(\alpha, \beta)} x-b_{i}^{(\alpha, \beta)}\right) \mathscr{P}_{i}^{(\alpha, \beta)}(x)-c_{i}^{(\alpha, \beta)} \mathscr{P}_{i-1}^{(\alpha, \beta)}(x), \quad i \geq 1, \\
& \mathscr{P}_{0}^{(\alpha, \beta)}(x)=1, \quad \mathscr{P}_{1}^{(\alpha, \beta)}(x)=\frac{1}{2}(\alpha+\beta+2) x+\frac{1}{2}(\alpha-\beta),
\end{aligned}
$$

where

$$
\begin{aligned}
& a_{i}^{(\alpha, \beta)}=\frac{(2 i+\alpha+\beta+1)(2 i+\alpha+\beta+2)}{2(i+1)(i+\alpha+\beta+1)}, \\
& b_{i}^{(\alpha, \beta)}=\frac{(2 i+\alpha+\beta+1)\left(\beta^{2}-\alpha^{2}\right)}{2(i+1)(i+\alpha+\beta+1)(2 i+\alpha+\beta)}, \\
& c_{i}^{(\alpha, \beta)}=\frac{(2 i+\alpha+\beta+2)(i+\alpha)(i+\beta)}{(i+1)(i+\alpha+\beta+1)(2 i+\alpha+\beta)} .
\end{aligned}
$$

The value of Jacobi polynomial at $x=1$ and $x=-1$ is:

$$
\begin{aligned}
& \mathscr{P}_{i}^{(\alpha, \beta)}(1)=\frac{\Gamma(i+\alpha+1)}{i !(\alpha+1)}, \\
& \mathscr{P}_{i}^{(\alpha, \beta)}(-1)=(-1)^{i} \frac{\Gamma(i+\beta+1)}{i !(\beta+1)} .
\end{aligned}
$$

The Legendre polynomial is $L_{n}(x)=\mathscr{P}_{n}^{(0,0)}$ and the Chebyshev polynomial is $T_{n}(x)=$ $\mathscr{P}_{n}^{\left(-\frac{1}{2},-\frac{1}{2}\right)}$. So it makes sense to study the general Jacobi polynomials.

Now, we give the analytic form of Jacobi polynomials as follows:

$$
\begin{aligned}
\mathscr{P}_{j}^{(\alpha, \beta)}(x) & =\sum_{k=0}^{j} \frac{(-1)^{j-k} \Gamma(j+1+\beta) \Gamma(j+1+k+\alpha+\beta)}{\Gamma(k+\beta+1) \Gamma(j+1+\alpha+\beta)(j-k) ! k ! 2^{k}}(x+1)^{k} \\
& =\sum_{k=0}^{j} \frac{\Gamma(j+1+\alpha) \Gamma(j+1+k+\alpha+\beta)}{\Gamma(k+\alpha+1) \Gamma(j+1+\alpha+\beta)(j-k) ! k ! 2^{k}}(x-1)^{k} .
\end{aligned}
$$


In addition, the $p$-th derivative of polynomials reads:

$$
\partial_{x}^{(p)} \mathscr{P}_{j}^{(\alpha, \beta)}(x)=\frac{\Gamma(j+\alpha+\beta+p+1)}{2^{p} \Gamma(j+\alpha+\beta+1)} \mathscr{P}_{j-p}^{(\alpha+p, \beta+p)}(x),
$$

and we define the weighted space $L_{\omega^{(\alpha, \beta)}}^{2}$, the norm and the inner product as follows:

$$
(u, v)_{\omega^{(\alpha, \beta)}}=\int_{-1}^{1} u(x) v(x) \omega^{(\alpha, \beta)}(x) d x, \quad\|u\|_{L_{\omega^{(\alpha, \beta)}}^{2}}=(u, u)_{\omega^{(\alpha, \beta)}}^{\frac{1}{2}} .
$$

Then, we use the Jacobi-Gauss (JG) quadrature to approximate the integrals Eq. (2.6). Let $P_{N}$ be the set of polynomials whose highest degree not more than $N$. It follows that for all $u(x) \in P_{2 N}$, there exists a unique weights $\omega_{j}^{(\alpha, \beta)}$, such that

$$
\int_{-1}^{1} \omega^{(\alpha, \beta)}(x) u(x) d x=\sum_{j=0}^{N} \omega_{j}^{(\alpha, \beta)} u\left(x_{j}\right),
$$

where $x_{j}$ and $\omega_{j}^{(\alpha, \beta)},(j=0,1, \cdots, N)$ are used as usual to denote the nodes and the corresponding Christoffel numbers in the interval $[-1,1]$, respectively.

Theorem 2.1 (Jacobi-Gauss-Type Quadratures, [17]). The Jacobi-Gauss quadrature formula (2.7) is exact for any $u(x) \in P_{2 N+1}$ with the Jacobi-Gauss nodes $x_{j},(j=0,1, \cdots, N)$ being the zeros of $\mathscr{P}_{N+1}^{(\alpha, \beta)}(x)$ and the corresponding weights $\omega_{j}^{(\alpha, \beta)},(j=0,1, \cdots, N)$ given by

$$
\omega_{j}^{(\alpha, \beta)}=\frac{G_{N}^{\alpha, \beta}}{\mathscr{P}_{N}^{(\alpha, \beta)}\left(x_{j}\right) \partial_{x} \mathscr{P}_{N+1}^{(\alpha, \beta)}\left(x_{j}\right)},
$$

where

$$
G_{N}^{\alpha, \beta}=\frac{2^{\alpha+\beta}(2 N+\alpha+\beta+2) \Gamma(N+\alpha+1) \Gamma(N+\beta+1)}{(N+1) ! \Gamma(N+\alpha+\beta+2)} .
$$

Theorem 2.2 (Jacobi-Gauss-Radau Quadratures, [17]). The Jacobi-Gauss quadrature formula (2.7) is exact for any $u(x) \in P_{2 N}$ with $x_{0}=-1$ and $x_{j},(j=1, \cdots, N)$ be the zeros of $\mathscr{P}_{N}^{(\alpha, \beta+1)}(x)$ and the weights are given by

$$
\begin{aligned}
& \omega_{0}^{(\alpha, \beta)}=\frac{2^{\alpha+\beta+1}(\beta+1) \Gamma^{2}(\beta+1) N ! \Gamma(N+\alpha+1)}{\Gamma(N+\beta+2) \Gamma(N+\alpha+\beta+2)}, \\
& \omega_{j}^{(\alpha, \beta)}=\frac{1}{1+x_{j}} \frac{G_{N-1}^{\alpha, \beta+1}}{\mathscr{P}_{N-1}^{(\alpha, \beta+1)}\left(x_{j}\right) \partial_{x} \mathscr{P}_{N}^{(\alpha, \beta+1)}\left(x_{j}\right)}, \quad 1 \leq j \leq N,
\end{aligned}
$$

where the constants $G_{N}^{\alpha, \beta}$ are defined in (2.8). 
Theorem 2.3 (Jacobi-Gauss-Lobatto Quadratures, [17]). The Jacobi-Gauss quadrature formula (2.7) is exact for any $u(x) \in P_{2 N-1}$. Let $x_{0}=-1, x_{N}=1$, and $x_{j},(j=1, \cdots, N-1)$ are the zeros of $\partial_{x} \mathscr{P}_{N}^{(\alpha, \beta)}(x)$, the corresponding weights are given by:

$$
\begin{aligned}
& \omega_{0}^{(\alpha, \beta)}=\frac{2^{\alpha+\beta+1}(\beta+1) \Gamma^{2}(\beta+1) \Gamma(N) \Gamma(N+\alpha+1)}{\Gamma(N+\beta+1) \Gamma(N+\alpha+\beta+2)}, \\
& \omega_{N}^{(\alpha, \beta)}=\frac{2^{\alpha+\beta+1}(\alpha+1) \Gamma^{2}(\alpha+1) \Gamma(N) \Gamma(N+\beta+1)}{\Gamma(N+\alpha+1) \Gamma(N+\alpha+\beta+2)}, \\
& \omega_{j}^{(\alpha, \beta)}=\frac{1}{1-x_{j}^{2}} \frac{G_{N-2}^{\alpha+1, \beta+1}}{\mathscr{P}_{N-2}^{(\alpha+1, \beta+1)}\left(x_{j}\right) \partial_{x} \mathscr{P}_{N-1}^{(\alpha+1, \beta+1)}\left(x_{j}\right)}, \quad 1 \leq j \leq N-1,
\end{aligned}
$$

where the constants $G_{N}^{\alpha, \beta}$ are defined in (2.8).

Next, suppose a function $u(x)$ which is infinitely differentiable in $[-1,1]$, then it can be written in terms as Jacobi polynomials:

$$
u(x)=\sum_{j=0}^{\infty} c_{j} \mathscr{P}_{j}^{(\alpha, \beta)}(x),
$$

and the coefficients $c_{j}$ as follows:

$$
c_{j}=\frac{1}{h_{j}^{(\alpha, \beta)}} \int_{-1}^{1} u(x) \mathscr{P}_{j}^{(\alpha, \beta)}(x) \omega^{(\alpha, \beta)}(x) d x, \quad j=0,1,2, \cdots
$$

In reality, we usually consider the first $N+1$ terms of the Jacobi polynomials for obtain an approximate expression of the function $u(x)$. Therefore $u(x)$ can be written as follows:

$$
u(x) \approx \sum_{j=0}^{N} c_{j} \mathscr{P}_{j}^{(\alpha, \beta)}(x) .
$$

\section{JGLC method for space discretization of FGLE}

In this section, we propose collocation method to solving the fractional Ginzburg-Landau equations in one- and two-dimension. The proposed scheme mainly depend on the JGLC method. We discretize the FGLE in the spatial direction by using the Jacobi-GaussLobatto (JGL) interpolation nodes to convert the FGLE into a system of ODEs.

\subsection{Discretization in one-dimensional}

In this subsection, we purpose to use a JGLC method for one-dimensional FGLE numerical solutions. In the process of JGLC method, we will chose the JGL quadrature nodes as 
the interpolation points. First, we want to acquire high accuracy that these interpolation points are usually related to Gauss integration formula, see [18]. Then, the distribution of these points in $[-1,1]$ and when we choose the nodes of JGL quadrature, the initial conditions will be satisfied automatically at these interpolations points, we no need other equations to solve these initial conditions. Therefore, the JGLC method is more reasonable.

We consider the FGLE in the following form:

$$
u_{t}+(v+i \eta)(-\Delta)^{\frac{a}{2}} u+(k+i \zeta)|u|^{2} u-\gamma u=0,
$$

where $1<a<2, x \in(-1,1), t \in(0, T], v>0, k>0, \eta, \zeta, \gamma$ are given real constants and the initial-boundary conditions:

$$
\begin{array}{ll}
u(x, 0)=u_{0}(x), & x \in(-1,1), \\
u(-1, t)=u(1, t)=0, & t \in(0, T] .
\end{array}
$$

Due to the numerical solution $u(x, t)$ is an complex function, we decompose it into its real and imaginary parts:

$$
u(x, t)=f(x, t)+i g(x, t), \quad u_{0}(x)=\xi_{1}(x)+i \xi_{2}(x),
$$

where $f(x, t), g(x, t), \xi_{1}(x)$ and $\xi_{2}(x)$ are real functions.

Let us denote $f_{t}=f_{t}(x, t), f=f(x, t), g_{t}=g_{t}(x, t)$, and $g=g(x, t)$. We apply the Eq. (3.3) and Eq. (3.1) to get

$$
f_{t}+i g_{t}+(v+i \eta)(-\Delta)^{\frac{\alpha}{2}}(f+i g)+(k+i \zeta)\left(f^{2}+g^{2}\right)(f+i g)-\gamma(f+i g)=0,
$$

and

$$
\begin{aligned}
& i\left(g_{t}+v(-\Delta)^{\frac{a}{2}} g+\eta(-\Delta)^{\frac{a}{2}} f+k\left(f^{2}+g^{2}\right) g+\zeta\left(f^{2}+g^{2}\right) f-\gamma g\right) \\
& \quad+f_{t}+v(-\Delta)^{\frac{a}{2}} f-\eta(-\Delta)^{\frac{a}{2}} g+k\left(f^{2}+g^{2}\right) f-\zeta\left(f^{2}+g^{2}\right) g-\gamma f=0 .
\end{aligned}
$$

Eq. (3.5) can be written as two coupled nonlinear fractional partial differential equations:

$$
\begin{aligned}
& f_{t}+v(-\Delta)^{\frac{a}{2}} f-\eta(-\Delta)^{\frac{a}{2}} g+k\left(f^{2}+g^{2}\right) f-\zeta\left(f^{2}+g^{2}\right) g-\gamma f=0, \\
& g_{t}+v(-\Delta)^{\frac{a}{2}} g+\eta(-\Delta)^{\frac{a}{2}} f+k\left(f^{2}+g^{2}\right) g+\zeta\left(f^{2}+g^{2}\right) f-\gamma g=0,
\end{aligned}
$$

with the initial-boundary conditions:

$$
\begin{array}{ll}
f(x, 0)=\xi_{1}(x), \quad g(x, 0)=\xi_{2}(x), & x \in(-1,1), \\
f(-1, t)=f(1, t)=0, & t \in(0, T], \\
g(-1, t)=g(1, t)=0, & t \in(0, T] .
\end{array}
$$


We write the approximate solution of $f(x, t)$ and $g(x, t)$ in the form of the Jacobi polynomials:

$$
\begin{aligned}
& f(x, t)=\sum_{j=0}^{N} a_{j}(t) \mathscr{P}_{j}^{(\alpha, \beta)}(x), \\
& g(x, t)=\sum_{j=0}^{N} b_{j}(t) \mathscr{P}_{j}^{(\alpha, \beta)}(x) .
\end{aligned}
$$

From Eq. (2.2) and Eq. (2.11), we obtain that

$$
\begin{aligned}
& a_{j}(t)=\frac{1}{h_{j}^{(\alpha, \beta)}} \int_{-1}^{1} f(x, t) \mathscr{P}_{j}^{(\alpha, \beta)}(x) \omega^{(\alpha, \beta)}(x) d x, \\
& b_{j}(t)=\frac{1}{h_{j}^{(\alpha, \beta)}} \int_{-1}^{1} g(x, t) \mathscr{P}_{j}^{(\alpha, \beta)}(x) \omega^{(\alpha, \beta)}(x) d x .
\end{aligned}
$$

Let us denote

$$
f_{i}(t)=f\left(x_{i}, t\right), \quad g_{i}(t)=g\left(x_{i}, t\right) .
$$

From the property of the Jacobi-Gauss quadrature (2.7) and Theorem 2.3, the coefficients $a_{j}(t)$ and $b_{j}(t)$ can be written as follows:

$$
\begin{aligned}
& a_{j}(t)=\frac{1}{h_{j}^{(\alpha, \beta)}} \sum_{i=0}^{N} \mathscr{P}_{j}^{(\alpha, \beta)}\left(x_{i}\right) \omega_{i}^{(\alpha, \beta)} f_{i}(t), \\
& b_{j}(t)=\frac{1}{h_{j}^{(\alpha, \beta)}} \sum_{i=0}^{N} \mathscr{P}_{j}^{(\alpha, \beta)}\left(x_{i}\right) \omega_{i}^{(\alpha, \beta)} g_{i}(t) .
\end{aligned}
$$

Employing Eqs. (3.9) and (3.10), the approximate solutions of Eq. (3.8b) can be written as follws:

$$
\begin{aligned}
& f(x, t)=\sum_{i=0}^{N}\left(\sum_{j=0}^{N} \frac{1}{h_{j}^{(\alpha, \beta)}} \mathscr{P}_{j}^{(\alpha, \beta)}\left(x_{i}\right) \omega_{i}^{(\alpha, \beta)} \mathscr{P}_{j}^{(\alpha, \beta)}(x)\right) f_{i}(t), \\
& g(x, t)=\sum_{i=0}^{N}\left(\sum_{j=0}^{N} \frac{1}{h_{j}^{(\alpha, \beta)}} \mathscr{P}_{j}^{(\alpha, \beta)}\left(x_{i}\right) \omega_{i}^{(\alpha, \beta)} \mathscr{P}_{j}^{(\alpha, \beta)}(x)\right) g_{i}(t) .
\end{aligned}
$$

Due to

$$
\begin{aligned}
{ }_{-1}^{R} D_{x}^{a}(x+1)^{k} & =\frac{1}{\Gamma(2-a)} \frac{d^{2}}{d x^{2}} \int_{-1}^{x}(x-s)^{1-a}(s+1)^{k} d s \\
& =\frac{\Gamma(k+1)}{\Gamma(k+1-a)}(x+1)^{k-a},
\end{aligned}
$$


and Eq. (2.4), the left Riemann-Liouville fractional derivative of $f(x, t)$ is as follows:

$$
\begin{aligned}
{ }_{-1}^{R} D_{x}^{a} f(x, t) & =\sum_{i=0}^{N}\left(\sum_{j=0}^{N} \frac{1}{h_{j}^{(\alpha, \beta)}} \mathscr{P}_{j}^{(\alpha, \beta)}\left(x_{i}\right) \omega_{i}^{(\alpha, \beta)}{ }_{-1}^{R} D_{x}^{a}\left[\mathscr{P}_{j}^{(\alpha, \beta)}(x)\right]\right) f_{i}(t) \\
& =\sum_{i=0}^{N}\left(\sum_{j=0}^{N} \frac{1}{h_{j}^{(\alpha, \beta)}} \mathscr{P}_{j}^{(\alpha, \beta)}\left(x_{i}\right) \omega_{i}^{(\alpha, \beta)} P_{L, j}^{(\alpha, \beta)}(x)\right) f_{i}(t) \\
& =\sum_{i=0}^{N}{ }_{L} D_{i}(x) f_{i}(t),
\end{aligned}
$$

where

$$
\begin{aligned}
{ }_{L} D_{i}(x) & =\sum_{j=0}^{N} \frac{1}{h_{j}^{(\alpha, \beta)}} \mathscr{P}_{j}^{(\alpha, \beta)}\left(x_{i}\right) \omega_{i}^{(\alpha, \beta)} P_{L, j}^{(\alpha, \beta)}(x), \\
P_{L, j}^{(\alpha, \beta)}(x) & =\sum_{k=0}^{j} \frac{(-1)^{j-k} \Gamma(j+1+\beta) \Gamma(j+1+k+\alpha+\beta)}{\Gamma(k+1+\beta) \Gamma(j+1+\alpha+\beta)(j-k) ! k ! 2^{k}-1} D_{x}^{a}(x+1)^{k} \\
& =\sum_{k=0}^{j} \frac{(-1)^{j-k} \Gamma(j+1+\beta) \Gamma(j+1+k+\alpha+\beta) \Gamma(1+k)(x+1)^{k-a}}{\Gamma(k+1+\beta) \Gamma(j+1+\alpha+\beta)(j-k) ! k ! 2^{k} \Gamma(k+1-a)} .
\end{aligned}
$$

Due to

$$
\begin{aligned}
{ }_{x}^{R} D_{1}^{a}(x-1)^{k} & =\frac{(-1)^{k}}{\Gamma(2-a)} \frac{d}{d x^{2}} \int_{x}^{1}(s-x)^{1-a}(1-x)^{k} d s \\
& =\frac{(-1)^{k} \Gamma(k+1)}{\Gamma(k+1-a)}(1-x)^{k-a} .
\end{aligned}
$$

Then the right Riemann-Liouville fractional of $f(x, t)$ as follow:

$$
\begin{aligned}
{ }_{x}^{R} D_{1}^{\alpha} f(x, t) & =\sum_{i=0}^{N}\left(\sum_{j=0}^{N} \frac{1}{h_{j}^{(\alpha, \beta)}} \mathscr{P}_{j}^{(\alpha, \beta)}\left(x_{i}\right) \omega_{i}^{(\alpha, \beta)} P_{R, j}^{(\alpha, \beta)}(x)\right) f_{i}(t) \\
& =\sum_{i=0}^{N}{ }_{R} D_{i}(x) f_{i}(t)
\end{aligned}
$$

where

$$
\begin{aligned}
& { }_{R} D_{i}(x)=\sum_{j=0}^{N} \frac{1}{h_{j}^{(\alpha, \beta)}} \mathscr{P}_{j}^{(\alpha, \beta)}\left(x_{i}\right) \omega_{i}^{(\alpha, \beta)} P_{R, j}^{(\alpha, \beta)}(x), \\
& P_{R, j}^{(\alpha, \beta)}(x)=\sum_{k=0}^{j} \frac{(-1)^{k} \Gamma(j+1+\alpha) \Gamma(j+1+k+\alpha+\beta) \Gamma(k+1)(1-x)^{k-a}}{\Gamma(k+\alpha+1) \Gamma(j+1+\alpha+\beta)(j-k) ! k ! 2^{k} \Gamma(k+1-a)} .
\end{aligned}
$$


By Eq. (1.4), the Riesz fractional derivative of $f(x, t)$ can be expressed by Eq. (3.13) and Eq. (3.16) as follows:

$$
\frac{\partial^{a}}{\partial|x|^{a}} f(x, t)=\sum_{i=0}^{N} \mathfrak{D}_{i}(x) f_{i}(t)
$$

where

$$
\mathfrak{D}_{i}(x)=-\frac{1}{2 \cos \left(\frac{\pi a}{2}\right)}\left[{ }_{L} D_{i}(x)+{ }_{R} D_{i}(x)\right] .
$$

By the same method

$$
\frac{\partial^{a}}{\partial|x|^{a}} g(x, t)=\sum_{i=0}^{N} \mathfrak{D}_{i}(x) g_{i}(t),
$$

where

$$
\mathfrak{D}_{i}(x)=-\frac{1}{2 \cos \left(\frac{\pi a}{2}\right)}\left[{ }_{L} D_{i}(x)+{ }_{R} D_{i}(x)\right] .
$$

Employing Eqs. (3.11)-(3.21), Eq. (3.6) can be written as follows:

$$
\begin{aligned}
& f_{t}-v \sum_{i=0}^{N} \mathfrak{D}_{i}(x) f_{i}(t)+\eta \sum_{i=0}^{N} \mathfrak{D}_{i}(x) g_{i}(t)+\left(f^{2}+g^{2}\right)(k f-\zeta g)-\gamma f=0, \\
& g_{t}-v \sum_{i=0}^{N} \mathfrak{D}_{i}(x) g_{i}(t)-\eta \sum_{i=0}^{N} \mathfrak{D}_{i}(x) f_{i}(t)+\left(f^{2}+g^{2}\right)(k g+\zeta f)-\gamma g=0 .
\end{aligned}
$$

Let us denote

$$
\dot{f}(t)=\frac{\partial}{\partial t} f(x, t), \quad \dot{g}(t)=\frac{\partial}{\partial t} g(x, t) .
$$

Now, we use the collocation method to convert Eq. (3.22) with its initial-boundary conditions into a system of ODEs in the time variable. From Theorem 2.3, supposing $\left\{x_{i}\right\}$ are the JGL interpolation nodes and applying these nodes in Eq. (3.22), Eq. (3.22) provides a system of $2(N+1)$ ODEs:

$$
\begin{aligned}
\dot{f}_{n}(t)=v \sum_{i=0}^{N} \mathfrak{D}_{i}\left(x_{n}\right) f_{i}(t)-\eta \sum_{i=0}^{N} \mathfrak{D}_{i}\left(x_{n}\right) g_{i}(t) & \\
\quad & -\left(f_{n}^{2}(t)+g_{n}^{2}(t)\right)\left(k f_{n}(t)-\zeta g_{n}(t)\right)+\gamma f_{n}(t), \\
\dot{g}_{n}(t)=v \sum_{i=0}^{N} \mathfrak{D}_{i}\left(x_{n}\right) g_{i}(t)+\eta \sum_{i=0}^{N} \mathfrak{D}_{i}\left(x_{n}\right) f_{i}(t) & \\
& \quad-\left(f_{n}^{2}(t)+g_{n}^{2}(t)\right)\left(k g_{n}(t)+\zeta f_{n}(t)\right)+\gamma g_{n}(t), \quad n=0,1, \cdots, N,
\end{aligned}
$$


where $f_{0}(t)=f_{N}(t)=0, g_{0}(t)=g_{N}(t)=0$ and subject to the initial values:

$$
f_{n}(0)=\xi_{1}\left(x_{n}\right), \quad g_{n}(0)=\xi_{2}\left(x_{n}\right), \quad n=0,1, \cdots N .
$$

We will discuss a new numerical method to solve the above linear system of ODEs in Section 4.

\subsection{Discretization in two-dimension}

In this section, we also use the JGLC method to discretize the two-dimensional FGLE. It has the following form:

$$
\begin{aligned}
& \frac{\partial u(x, y, t)}{\partial t}+\left((v+i \eta)\left(-\frac{\partial^{a}}{\partial|x|^{a}}-\frac{\partial^{a}}{\partial|y|^{a}}\right)\right. \\
& \left.+(k+i \zeta)|u(x, y, t)|^{2}-\gamma\right) u(x, y, t)=0, \quad(x, y) \in \partial \Omega, \quad t \in(0, T]
\end{aligned}
$$

with the initial and boundary conditions:

$$
\begin{array}{ll}
u(x, y, 0)=u_{0}(x, y), & (x, y) \in \Omega, \\
u(x, y, t)=0, & (x, y) \in \partial \Omega, \quad t \in(0, T],
\end{array}
$$

where $u(x, y, t)$ is an unknown complex function, $1<a<2, v>0, k>0, \eta, \zeta$ and $\gamma$ are given real constants. $\Omega=(-1,1) \times(-1,1)$ and $\partial \Omega$ is the boundary of $\Omega$.

We first write the unknown complex functions $u(x, y, t)$ and $u_{0}(x, y)$ in terms of real and imaginary parts as follows:

$$
u(x, y, t)=f(x, y, t)+i g(x, y, t), \quad u_{0}(x, y)=\xi_{1}(x, y)+i \xi_{2}(x, y),
$$

where $f(x, y, t), g(x, y, t), \xi_{1}(x, y)$ and $\xi_{2}(x, y)$ are real functions. Substituting Eq. (3.27) in Eq. (3.25) and Eq. (3.26), the fractional partial differential equations read as follows:

$$
\begin{gathered}
\frac{\partial}{\partial t}(f(x, y, t)+i g(x, y, t))+(v+i \eta)\left(-\frac{\partial^{a}}{\partial|x|^{\alpha}}-\frac{\partial^{a}}{\partial|y|^{a}}\right)(f(x, y, t)+i g(x, y, t)) \\
+\left((k+i \zeta)\left(f^{2}(x, y, t)+g^{2}(x, y, t)\right)-\gamma\right)(f(x, y, t)+i g(x, y, t))=0 .
\end{gathered}
$$

Then, the above equation can be written as:

$$
\begin{gathered}
\frac{\partial}{\partial t} f(x, y, t)+v\left(-\frac{\partial^{a}}{\partial|x|^{a}}-\frac{\partial^{a}}{\partial|y|^{a}}\right) f(x, y, t)-\eta\left(-\frac{\partial^{\alpha}}{\partial|x|^{a}}-\frac{\partial^{a}}{\partial|y|^{a}}\right) g(x, y, t) \\
+(k f(x, y, t)-\zeta g(x, y, t))\left(f^{2}(x, y, t)+g^{2}(x, y, t)\right)-\gamma f(x, y, t)=0, \\
\frac{\partial}{\partial t} g(x, y, t)+v\left(-\frac{\partial^{a}}{\partial|x|^{a}}-\frac{\partial^{a}}{\partial|y|^{a}}\right) g(x, y, t)+\eta\left(-\frac{\partial^{a}}{\partial|x|^{a}}-\frac{\partial^{a}}{\partial|y|^{a}}\right) f(x, y, t) \\
+(k g(x, y, t)+\zeta f(x, y, t))\left(f^{2}(x, y, t)+g^{2}(x, y, t)\right)-\gamma g(x, y, t)=0,
\end{gathered}
$$


with initial-boundary conditions:

$$
\begin{array}{lll}
f(x, y, 0)=\xi_{1}(x, y), \quad g(x, y, 0)=\xi_{2}(x, y), & (x, y) \in \Omega, & \\
f(-1, y, t)=f(1, y, t)=0, & y \in(-1,1), & t \in(0, T], \\
f(x,-1, t)=f(x, 1, t)=0, & x \in(-1,1), & t \in(0, T], \\
g(-1, y, t)=g(1, y, t)=0, & y \in(-1,1), & t \in(0, T], \\
g(x,-1, t)=g(x, 1, t)=0, & x \in(-1,1), & t \in(0, T] .
\end{array}
$$

Next, we convert the above coupled nonlinear fractional partial differential equations with its initial conditions into a system of ODEs based on the JGLC discretization.

Let the numerical solution be a double Jacobi polynomial series:

$$
\begin{aligned}
& f(x, y, t)=\sum_{i=0}^{N} \sum_{j=0}^{M} a_{i, j}(t) \mathscr{P}_{i}^{(\alpha, \beta)}(x) \mathscr{P}_{j}^{(\alpha, \beta)}(y), \\
& g(x, y, t)=\sum_{i=0}^{N} \sum_{j=0}^{M} b_{i, j}(t) \mathscr{P}_{i}^{(\alpha, \beta)}(x) \mathscr{P}_{j}^{(\alpha, \beta)}(y) .
\end{aligned}
$$

Let $\left\{x_{i}: 0 \leq i \leq N\right\}$ and $\left\{y_{j}: 0 \leq j \leq M\right\}$ be the JGL interpolation nodes of Jacobi polynomials $\mathscr{P}_{N}^{(\alpha, \beta)}(x)$ and $\mathscr{P}_{M}^{(\alpha, \beta)}(y)$ respectively. From the Jacobi Gauss quadrature (2.7) and Theorem 2.3, the coefficients $a_{i, j}(t), b_{i, j}(t)$ above are determined as follows:

$$
\begin{aligned}
& a_{i, j}(t)=\frac{1}{h_{i}^{(\alpha, \beta)} h_{j}^{(\alpha, \beta)}} \sum_{p=0}^{N} \sum_{q=0}^{M}\left(\mathscr{P}_{j}^{(\alpha, \beta)}\left(y_{q}\right) \omega_{q}^{(\alpha, \beta)} \mathscr{P}_{i}^{(\alpha, \beta)}\left(x_{p}\right) \omega_{p}^{(\alpha, \beta)}\right) f\left(x_{p}, y_{q}, t\right), \\
& b_{i, j}(t)=\frac{1}{h_{i}^{(\alpha, \beta)} h_{j}^{(\alpha, \beta)}} \sum_{p=0}^{N} \sum_{q=0}^{M}\left(\mathscr{P}_{j}^{(\alpha, \beta)}\left(y_{q}\right) \omega_{q}^{(\alpha, \beta)} \mathscr{P}_{i}^{(\alpha, \beta)}\left(x_{p}\right) \omega_{p}^{(\alpha, \beta)}\right) g\left(x_{p}, y_{q}, t\right) .
\end{aligned}
$$

For simplicity of the formula below, denote

$$
f\left(x_{p}, y_{q}, t\right)=f_{p, q}(t), \quad g\left(x_{p}, y_{q}, t\right)=g_{p, q}(t),
$$

then the approximate solutions Eq. (3.30) can be written as follows:

$$
\begin{aligned}
f(x, y, t)=\sum_{i=0}^{N} \sum_{j=0}^{M} \sum_{p=0}^{N} \sum_{q=0}^{M} \frac{\left(\mathscr{P}_{j}^{(\alpha, \beta)}\left(y_{q}\right) \omega_{q}^{(\alpha, \beta)} \mathscr{P}_{i}^{(\alpha, \beta)}\left(x_{p}\right) \omega_{p}^{(\alpha, \beta)}\right)}{h_{i}^{(\alpha, \beta)} h_{j}^{(\alpha, \beta)}} \\
\quad \times \mathscr{P}_{i}^{(\alpha, \beta)}(x) \mathscr{P}_{j}^{(\alpha, \beta)}(y) f_{p, q}(t), \\
g(x, y, t)=\sum_{i=0}^{N} \sum_{j=0}^{M} \sum_{p=0}^{N} \sum_{q=0}^{M} \frac{\left(\mathscr{P}_{j}^{(\alpha, \beta)}\left(y_{q}\right) \omega_{q}^{(\alpha, \beta)} \mathscr{P}_{i}^{(\alpha, \beta)}\left(x_{p}\right) \omega_{p}^{(\alpha, \beta)}\right)}{h_{i}^{(\alpha, \beta)} h_{h, j}^{(\alpha, \beta)}} \\
\quad \times \mathscr{P}_{i}^{(\alpha, \beta)}(x) \mathscr{P}_{j}^{(\alpha, \beta)}(y) g_{p, q}(t) .
\end{aligned}
$$


Then the left and right Riemann-Liouville fractional derivative of $f(x, y, t)$ as follows:

$$
\begin{aligned}
& { }_{-1}^{R} D_{x}^{a} f(x, y, t)=\sum_{i=0}^{N} \sum_{j=0}^{M} a_{i, j}(t) P_{L, i}^{(\alpha, \beta)}(x) \mathscr{P}_{j}^{(\alpha, \beta)}(y), \\
& { }_{-1}^{R} D_{y}^{a} f(x, y, t)=\sum_{i=0}^{N} \sum_{j=0}^{M} a_{i, j}(t) P_{L, j}^{(\alpha, \beta)}(y) \mathscr{P}_{i}^{(\alpha, \beta)}(x), \\
& { }_{x}^{R} D_{1}^{a} f(x, y, t)=\sum_{i=0}^{N} \sum_{j=0}^{M} a_{i, j}(t) P_{R, i}^{(\alpha, \beta)}(x) \mathscr{P}_{j}^{(\alpha, \beta)}(y), \\
& { }_{y}^{R} D_{1}^{a} f(x, y, t)=\sum_{i=0}^{N} \sum_{j=0}^{M} a_{i, j}(t) P_{R, j}^{(\alpha, \beta)}(y) \mathscr{P}_{i}^{(\alpha, \beta)}(x),
\end{aligned}
$$

where

$$
\begin{aligned}
& P_{L, i}^{(\alpha, \beta)}(x)=\sum_{p=0}^{i} \frac{(-1)^{i-p} \Gamma(i+1+\beta) \Gamma(i+1+p+\alpha+\beta) \Gamma(1+p)(x+1)^{p-a}}{\Gamma(p+\beta+1) \Gamma(i+1+\alpha+\beta)(i-p) ! p ! 2^{p} \Gamma(1+p-a)}, \\
& P_{L, j}^{(\alpha, \beta)}(y)=\sum_{q=0}^{j} \frac{(-1)^{j-q} \Gamma(j+1+\beta) \Gamma(j+1+q+\alpha+\beta) \Gamma(1+q)(y+1)^{q-a}}{\Gamma(q+\beta+1) \Gamma(j+1+\alpha+\beta)(j-q) ! q ! 2^{q} \Gamma(1+q-a)} \\
& P_{R, i}^{(\alpha, \beta)}(x)=\sum_{p=0}^{i} \frac{(-1)^{p} \Gamma(i+1+\alpha) \Gamma(i+1+p+\alpha+\beta) \Gamma(1+p)(1-x)^{p-a}}{\Gamma(p+\alpha+1) \Gamma(i+1+\alpha+\beta)(i-p) ! p ! 2^{p} \Gamma(1+p-a)} \\
& P_{R, j}^{(\alpha, \beta)}(y)=\sum_{q=0}^{j} \frac{(-1)^{q} \Gamma(j+1+\alpha) \Gamma(j+1+q+\alpha+\beta) \Gamma(1+q)(1-y)^{q-a}}{\Gamma(q+\alpha+1) \Gamma(j+1+\alpha+\beta)(j-q) ! q ! 2^{q} \Gamma(1+q-a)} .
\end{aligned}
$$

Let

$$
\begin{aligned}
& { }_{-1}^{R} D_{x}^{a} f(x, y, t)=\sum_{i=0}^{N} \sum_{j=0}^{M}{ }_{L} D_{i, j}(x, y) f_{i, j}(t), \\
& { }_{-1}^{R} D_{y}^{a} f(x, y, t)=\sum_{i=0}^{N} \sum_{j=0}^{M}{ }_{L} \bar{D}_{i, j}(x, y) f_{i, j}(t), \\
& { }_{x}^{R} D_{1}^{a} f(x, y, t)=\sum_{i=0}^{N} \sum_{j=0}^{M}{ }_{R} D_{i, j}(x, y) f_{i, j}(t), \\
& { }_{y}^{R} D_{1}^{a} f(x, y, t)=\sum_{i=0}^{N} \sum_{j=0}^{M}{ }_{R} \bar{D}_{i, j}(x, y) f_{i, j}(t),
\end{aligned}
$$

where

$$
{ }_{L} D_{i, j}(t)=\sum_{p=0}^{N} \sum_{q=0}^{M} \frac{\left(\mathscr{P}_{j}^{(\alpha, \beta)}\left(y_{q}\right) \omega_{q}^{(\alpha, \beta)} \mathscr{P}_{i}^{(\alpha, \beta)}\left(x_{p}\right) \omega_{p}^{(\alpha, \beta)}\right)}{h_{i} h_{j}} P_{L, i}^{(\alpha, \beta)}(x) \mathscr{P}_{j}^{(\alpha, \beta)}(y),
$$




$$
\begin{aligned}
& { }_{L} \bar{D}_{i, j}(t)=\sum_{p=0}^{N} \sum_{q=0}^{M} \frac{\left(\mathscr{P}_{j}^{(\alpha, \beta)}\left(y_{q}\right) \omega_{q}^{(\alpha, \beta)} \mathscr{P}_{i}^{(\alpha, \beta)}\left(x_{p}\right) \omega_{p}^{(\alpha, \beta)}\right)}{h_{i} h_{j}} P_{L, j}^{(\alpha, \beta)}(y) \mathscr{P}_{i}^{(\alpha, \beta)}(x), \\
& { }_{R} D_{i, j}(t)=\sum_{p=0}^{N} \sum_{q=0}^{M} \frac{\left(\mathscr{P}_{j}^{(\alpha, \beta)}\left(y_{q}\right) \omega_{q}^{(\alpha, \beta)} \mathscr{P}_{i}^{(\alpha, \beta)}\left(x_{p}\right) \omega_{p}^{(\alpha, \beta)}\right)}{h_{i} h_{j}} P_{R, i}^{(\alpha, \beta)}(x) \mathscr{P}_{j}^{(\alpha, \beta)}(y), \\
& { }_{R} \bar{D}_{i, j}(t)=\sum_{p=0}^{N} \sum_{q=0}^{M} \frac{\left(\mathscr{P}_{j}^{(\alpha, \beta)}\left(y_{q}\right) \omega_{h, M, q}^{(\alpha, \beta)} \mathscr{P}_{i}^{(\alpha, \beta)}\left(x_{p}\right) \omega_{p}^{(\alpha, \beta)}\right)}{h_{i} h_{j}} P_{R, j}^{(\alpha, \beta)}(y) \mathscr{P}_{i}^{(\alpha, \beta)}(x) .
\end{aligned}
$$

By the formula (1.4), the Riesz fractional derivative of $f(x, y, t)$ can be written with Eq. (3.36) as follows:

$$
\frac{\partial^{a}}{\partial|x|^{a}} f(x, y, t)=\sum_{i=0}^{N} \sum_{j=0}^{M} \mathfrak{D}_{i, j}(x, y) f_{i, j}(t),
$$

where

$$
\mathfrak{D}_{i, j}(x, y)=-\frac{1}{2 \cos \left(\frac{\pi a}{2}\right)}\left[{ }_{L} D_{i, j}(x, y)+{ }_{R} D_{i, j}(x, y)\right] .
$$

By the same method:

$$
\frac{\partial^{a}}{\partial|y|^{a}} g(x, y, t)=\sum_{i=0}^{N} \sum_{j=0}^{M} \overline{\mathfrak{D}}_{i, j}(x, y) g_{i, j}(t)
$$

where

$$
\overline{\mathfrak{D}}_{i, j}(x, y)=-\frac{1}{2 \cos \left(\frac{\pi a}{2}\right)}\left[{ }_{L} \bar{D}_{i, j} y(x, y)+{ }_{R} \bar{D}_{i, j}(x, y)\right] .
$$

Adopting Eqs. (3.38)-(3.41), Eq. (3.28) reads:

$$
\begin{aligned}
\dot{f}(x, y, t)=v\left(\sum_{i=0}^{N} \sum_{j=0}^{M} \mathfrak{D}_{i, j}(x, y) f_{i, j}(t)+\sum_{i=0}^{N} \sum_{j=0}^{M} \overline{\mathfrak{D}}_{i, j}(x, y) f_{i, j}(t)\right) \\
-\eta\left(\sum_{i=0}^{N} \sum_{j=0}^{M} \mathfrak{D}_{i, j}(x, y) g_{i, j}(t)+\sum_{i=0}^{N} \sum_{j=0}^{M} \overline{\mathfrak{D}}_{i, j}(x, y) g_{i, j}(t)\right) \\
\quad-(k f(x, y, t)-\zeta g(x, y, t))\left(f^{2}(x, y, t)+g^{2}(x, y, t)\right)+\gamma f(x, y, t), \\
\dot{g}(x, y, t)=v\left(\sum_{i=0}^{N} \sum_{j=0}^{M} \mathfrak{D}_{i, j}(x, y) g_{i, j}(t)+\sum_{i=0}^{N} \sum_{j=0}^{M} \overline{\mathfrak{D}}_{i, j}(x, y) g_{i, j}(t)\right) \\
+\eta\left(\sum_{i=0}^{N} \sum_{j=0}^{M} \mathfrak{D}_{i, j}(x, y) f_{i, j}(t)+\sum_{i=0}^{N} \sum_{j=0}^{M} \overline{\mathfrak{D}}_{i, j}(x, y) f_{i, j}(t)\right) \\
-(k g(x, y, t)+\zeta f(x, y, t))\left(f^{2}(x, y, t)+g^{2}(x, y, t)\right)-\gamma g(x, y, t) .
\end{aligned}
$$


Then, we convert Eq. (3.42) into a system of ODEs with the time variable based on collocation method with the JGL interpolation nodes $x_{n},(n=0,1, \cdots, N)$ of $\mathscr{P}_{N}^{(\alpha, \beta)}(x), y_{m}$, $(m=0,1, \cdots, M)$ of $\mathscr{P}_{M}^{(\alpha, \beta)}(y)$. Due to the initial condition (3.29), the above equation can be written as follows:

$$
\begin{array}{r}
\dot{f}_{n, m}(t)=v\left(\sum_{i=0}^{N} \sum_{j=0}^{M} \mathfrak{D}_{i, j}\left(x_{n}, y_{m}\right) f_{i, j}(t)+\sum_{i=0}^{N} \sum_{j=0}^{M} \overline{\mathfrak{D}}_{i, j}\left(x_{n}, y_{m}\right) f_{i, j}(t)\right) \\
-\eta\left(\sum_{i=0}^{N} \sum_{j=0}^{M} \mathfrak{D}_{i, j}\left(x_{n}, y_{m}\right) g_{i, j}(t)+\sum_{i=0}^{N} \sum_{j=0}^{M} \overline{\mathfrak{D}}_{i, j}\left(x_{n}, y_{m}\right) g_{i, j}(t)\right) \\
-\left(k f_{n, m}(t)-\zeta g_{n, m}(t)\right)\left(f_{n, m}^{2}(t)+g_{n, m}^{2}(t)\right)+\gamma f_{n, m}(t), \\
\dot{g}_{n, m}(t)=v\left(\sum_{i=0}^{N} \sum_{j=0}^{M} \mathfrak{D}_{i, j}\left(x_{n}, y_{m}\right) g_{i, j}(t)+\sum_{i=0}^{N} \sum_{j=0}^{M} \overline{\mathfrak{D}}_{i, j}\left(x_{n}, y_{m}\right) g_{i, j}(t)\right) \\
+\eta\left(\sum_{i=0}^{N} \sum_{j=0}^{M} \mathfrak{D}_{i, j}\left(x_{n}, y_{m}\right) f_{i, j}(t)+\sum_{i=0}^{N} \sum_{j=0}^{M} \overline{\mathfrak{D}}_{i, j}\left(x_{n}, y_{m}\right) f_{i, j}(t)\right) \\
-\left(k g_{n, m}(t)+\zeta f_{n, m}(t)\right)\left(f_{n, m}^{2}(t)+g_{n, m}^{2}(t)\right)-\gamma g_{n, m}(t), \\
n=0,1, \cdots, N, \quad m=0,1, \cdots, M,
\end{array}
$$

and subject initial conditions:

$$
f_{n, m}(0)=\xi_{1}\left(x_{n}, y_{m}\right), \quad g_{n, m}(0)=\xi_{2}\left(x_{n}, y_{m}\right), \quad n=0,1, \cdots, N, \quad m=0,1, \cdots, M .
$$

In addition, the values of $f_{0, q}(t), f_{N, q}(t), f_{p, 0}(t), f_{p, N}(t), g_{0, q}(t), g_{N, q}(t), g_{p, 0}(t), g_{p, N}(t)$ are computed as follows:

$$
\begin{aligned}
& f_{0, q}(t)=f_{N, q}(t)=0, \quad q=0, \cdots, M, \\
& f_{p, 0}(t)=f_{p, N}(t)=0, \quad p=0, \cdots, N \text {, } \\
& g_{0, q}(t)=g_{N, q}(t)=0, \quad q=0, \cdots, M \text {, } \\
& g_{p, 0}(t)=g_{p, N}(t)=0, \quad \quad p=0, \cdots, N \text {. }
\end{aligned}
$$

The above results are a system of $2(N \times M)$ ordinary differential equations. In the next section, we proposed a new method to solve the above system of ODEs.

\section{JGRC method for ODEs}

In this section, we propose a new method deal with the systems of ordinary differential equation obtained from the previous section, i.e., Eq. (3.23) and Eq. (3.24), Eq. (3.43) and Eq. (3.44), namely:

$$
\dot{\varphi}_{r}(t)=G_{r}\left(t, \varphi_{1}(t), \cdots, \varphi_{R}(t)\right), \quad r=1, \cdots, R, \quad t \in(0, T],
$$


and subject to

$$
\varphi_{r}(0)=g_{r}, \quad r=1, \cdots, R,
$$

where $G_{r}\left(t, \varphi_{1}(t), \cdots, \varphi_{r}(t)\right), r=1, \cdots, R$ are given nonlinear functions.

In this process, we will choose the JGR quadrature nodes as interpolation nodes for discretizing above ODEs. First, Gauss quadratures can provide a high accuracy. Then, we use the nodes of the JGR quadrature so that the initial conditions satisfied automatically in the collocation method.

First, let us expand the approximate solutions of $\varphi_{r}(t)$ by the Jacobi polynomials in the form:

$$
\varphi_{r}(t)=\sum_{j=0}^{K} a_{r, j} \mathscr{P}_{T, j}^{(\alpha, \beta)}(2 t-T), \quad r=1, \cdots, R .
$$

From the derivative relationship in Eq. (2.5), the first time derivative of the approximate solution is as follows:

$$
\dot{\varphi}_{r}(t)=\sum_{j=1}^{K} a_{r, j} \frac{\Gamma(j+\alpha+\beta+2)}{\Gamma(j+\alpha+\beta+1) T} \mathscr{P}_{T, j-1}^{(\alpha+1, \beta+1)}(2 t-T), \quad r=1, \cdots, R .
$$

Then, adopting Eqs. (4.3) and (4.4), Eqs. (4.1) and (4.2) can be written as follows :

$$
\begin{aligned}
& \sum_{j=1}^{K} a_{r, j} \frac{\Gamma(j+\alpha+\beta+2)}{\Gamma(j+\alpha+\beta+1) T} \mathscr{P}_{T, j-1}^{(\alpha+1, \beta+1)}(2 t-T) \\
= & G_{r}\left(t, \sum_{j=0}^{K} a_{1, j} \mathscr{P}_{T, j}^{(\alpha, \beta)}(2 t-T), \cdots, \sum_{j=0}^{K} a_{R, j} \mathscr{P}_{T, j}^{(\alpha, \beta)}(2 t-T)\right), \quad r=1, \cdots, R, \quad t \in(0, T],
\end{aligned}
$$

subject to

$$
\sum_{j=0}^{K} a_{r, j} \mathscr{P}_{T, j}^{(\alpha, \beta)}(-T)=g_{r}, \quad r=1, \cdots, R .
$$

Next, we discretize the time variable at $\left\{t_{j}\right\}$, based on JGRC method. Let $\left\{t_{j}\right\}$ be the JGR interpolation nodes, where $t_{0}=0$, and $t_{k},(k=1, \cdots, K)$ are zeros of $\mathscr{P}_{T, K}^{(\alpha, \beta+1)}(2 t-T)$. By Theorem 2.2, we get $(R \times K)$ algebraic equations:

$$
\begin{aligned}
& \sum_{j=1}^{K} a_{r, j} \frac{\Gamma(j+\alpha+\beta+2)}{\Gamma(j+\alpha+\beta+1)} \mathscr{P}_{T, j-1}^{(\alpha+1, \beta+1)}\left(2 t_{k}-T\right) \\
= & G_{r}\left(t_{k}, \sum_{j=0}^{K} a_{1, j} \mathscr{P}_{T, j}^{(\alpha, \beta)}\left(2 t_{k}-T\right), \cdots, \sum_{j=0}^{K} a_{R, j} \mathscr{P}_{T, j}^{(\alpha, \beta)}\left(2 t_{k}-T\right)\right), \\
& \quad r=1, \cdots, R, \quad k=1, \cdots, K .
\end{aligned}
$$


Because of the initial condition (4.6), we obtain another set of $R$ algebraic equations. Finally, combining two algebraic systems, we obtain $(R \times(K+1))$ algebraic equations for Jacobi expansion coefficient $a_{r, j}, r=1,2, \cdots, R, j=0,1, \cdots, K$. We would solve the system of equations by Newton's iteration method. Therefore, we can evaluate the approximate solutions (4.3).

\section{Error analysis}

In this section, we estimate the error of the numerical solution obtained by using JGLC method and JGRC method in solving the fractional Ginzburg-Landua equation. We bound the error in the sense of $L^{\infty}$ and $L^{2}$.

As $u(x, t)$ is a complex function, we decompose it into its real and imaginary parts:

$$
u(x, t)=f(x, t)+i g(x, t), \quad x \in[-1,1], \quad t \in[0, T],
$$

where $f(x, t)$ and $g(x, t)$ are smooth real functions.

Lemma 5.1 ([20]). If $\alpha, \beta>-1$, then

$$
\max _{-1 \leq x \leq 1}\left|\mathscr{P}_{n}^{\alpha, \beta}\right| \leq K n^{2}
$$

where $q=\max \left(\alpha, \beta,-\frac{1}{2}\right)$ and $K$ is a positive constant.

Let us define the discrete space by

$$
\mathcal{P}_{N, M}^{\alpha, \beta}=\operatorname{span}\left\{\mathscr{P}_{i}^{(\alpha, \beta)}(x) \mathscr{P}_{T, k}^{(\alpha, \beta)}(t), i=0,1, \cdots, M, k=0,1, \cdots, N\right\} .
$$

Let $f_{N, M}(x, t)$ and $g_{N, M}(x, t)$ be the numerical solutions approximating $f(x, t)$ and $g(x, t)$, respectively,

$$
f_{N, M}(x, t), g_{N, M}(x, t) \in \mathcal{P}_{N, M}^{\alpha, \beta}
$$

Let

$$
\begin{aligned}
& E_{1}(x, t)=\left|f(x, t)-f_{N, M}(x, t)\right|, \\
& E_{2}(x, t)=\left|g(x, t)-g_{N, M}(x, t)\right|, \\
& E_{3}(x, t)=\left|u(x, t)-u_{N, M}(x, t)\right|,
\end{aligned}
$$

where $u(x, t)=f(x, t)+i g(x, t)$ and $u_{N, M}(x, t)=f_{N, M}(x, t)+i g_{N, M}(x, t)$ are the exact solution and approximate solution, respectively. In particular,

$$
\begin{aligned}
E_{3}(x, t) & =\left|u(x, t)-u_{N, M}(x, t)\right|=\left|f(x, t)-f_{N, M}(x, t)+i\left(g(x, t)-g_{N, M}(x, t)\right)\right| \\
& =\sqrt{E_{1}^{2}(x, t)+E_{2}^{2}(x, t)} .
\end{aligned}
$$


Theorem 5.1. Let $u(x, t)=f(x, t)+i g(x, t)$ be the exact solution of the original continuous problem (3.1), which is sufficiently smooth. $u_{N, M}(x, t)=f_{N, M}(x, t)+i g_{N, M}(x, t)$ is the numerical solution obtained by (4.7). The following error estimate holds,

$$
\left\|u(x, t)-u_{N, M}(x, t)\right\|_{L^{\infty}} \leq\left(\left(C_{1}^{\prime} Q_{1}+C_{2}^{\prime} Q_{2}+C_{3}^{\prime} Q_{1} Q_{2}\right)^{2}+\left(H_{1}^{\prime} Q_{1}+H_{2}^{\prime} Q_{2}+H_{3}^{\prime} Q_{1} Q_{2}\right)^{2}\right)^{\frac{1}{2}},
$$

where

$$
Q_{1}=\frac{(M+1)^{q}}{(M+1) ! \kappa_{M}^{(\alpha, \beta)}}, \quad Q_{2}=\frac{(T / 2)^{N+1}(N+1)^{q}}{(N+1) ! \kappa_{N}^{(\alpha, \beta)}},
$$

and $C_{1}^{\prime}, C_{2}^{\prime}, C_{3}^{\prime}, H_{1}^{\prime}, H_{2}^{\prime}, H_{3}^{\prime}$ are constants, $q=\max \left(\alpha, \beta,-\frac{1}{2}\right)$ and $\kappa_{M}^{(\alpha, \beta)}$ is the coefficient of $\mathscr{P}_{M+1}^{(\alpha, \beta)}(x)$.

Proof. The maximum absolute error is defined by:

$$
\begin{aligned}
& M_{1}=\left\|E_{1}(x, t)\right\|_{L^{\infty}}=\max \left\{\left|E_{1}(x, t)\right|: \forall(x, t) \in[-1,1] \times[0, T]\right\}, \\
& M_{2}=\left\|E_{2}(x, t)\right\|_{L^{\infty}}=\max \left\{\left|E_{2}(x, t)\right|: \forall(x, t) \in[-1,1] \times[0, T]\right\}, \\
& M_{3}=\left\|E_{3}(x, t)\right\|_{L^{\infty}}=\max \left\{\left|E_{3}(x, t)\right|: \forall(x, t) \in[-1,1] \times[0, T]\right\},
\end{aligned}
$$

and clearly $M_{3} \leq \sqrt{M_{1}^{2}+M_{2}^{2}}$.

Because $f_{N, M}(x, t)$ is the best approximation in $L^{\infty}$-norm by the orthogonal expansion of $f(x, t)$ under the Jacobi polynomial basis, for any $v_{N, M}(x, t) \in \mathcal{P}_{N, M}^{\alpha, \beta}$, the following inequality holds:

$$
\left\|f(x, t)-f_{N, M}(x, t)\right\|_{L^{\infty}} \leq C\left\|f(x, t)-v_{N, M}(x, t)\right\|_{L^{\infty}},
$$

where $v_{N, M}(x, t)$ denotes the interpolating polynomial for $f(x, t)$ at $\left\{x_{i}\right\}$ (the JGL interpolation points of $\mathscr{P}_{M}^{(\alpha, \beta)}(x)$ ) and $\left\{t_{k}\right\}$ (the JGR interpolation of $\mathscr{P}_{T, K}^{(\alpha, \beta)}(t)$ ).

Thus,

$$
\begin{array}{r}
f(x, t)-v_{N, M}(x, t)=\frac{\partial^{M+1} f(\xi, t)}{(M+1) ! \partial x^{M+1}} \prod_{i=0}^{M}\left(x-x_{i}\right)+\frac{\partial^{N+1} f(x, \zeta)}{(N+1) ! \partial t^{N+1}} \prod_{i=0}^{N}\left(t-t_{k}\right) \\
-\frac{\partial^{M+N+2} f\left(\xi^{\prime}, \zeta^{\prime}\right)}{(M+1) !(N+1) ! \partial x^{M+1} \partial t^{N+1}} \prod_{i=0}^{M}\left(x-x_{i}\right) \prod_{i=0}^{N}\left(t-t_{k}\right),
\end{array}
$$

where $\xi, \xi^{\prime} \in[-1,1], \zeta, \zeta^{\prime} \in[0, T]$,

$$
\begin{aligned}
& \left\|f(x, t)-v_{N, M}(x, t)\right\|_{L^{\infty}} \\
& \leq \max _{(x, t) \in \Omega}\left|\frac{\partial^{M+1} f(\xi, t)}{\partial x^{M+1}}\right| \frac{\left\|\prod_{i=0}^{M}\left(x-x_{i}\right)\right\|_{L^{\infty}}}{(M+1) !}+\max _{(x, t) \in \Omega}\left|\frac{\partial^{N+1} f(x, \zeta)}{\partial t^{N+1}}\right| \frac{\left\|\prod_{i=0}^{N}\left(t-t_{k}\right)\right\|_{L^{\infty}}}{(N+1) !} \\
& \quad+\max _{(x, t) \in \Omega}\left|\frac{\partial^{M+N+2} f\left(\xi^{\prime}, \zeta^{\prime}\right)}{\partial x^{M+1} \partial t^{N+1}}\right| \frac{\left\|\prod_{i=0}^{M}\left(x-x_{i}\right)\right\|_{L^{\infty}}\left\|\prod_{i=0}^{N}\left(t-t_{k}\right)\right\|_{L^{\infty}}}{(M+1) !(N+1) !} .
\end{aligned}
$$


For the smooth function $f(x, t)$, there exist constants $C_{1}, C_{2}$ and $C_{3}$ such that

$$
\begin{aligned}
& \max _{(x, t) \in \Omega}\left|\frac{\partial^{M+1} f(\xi, t)}{\partial x^{M+1}}\right| \leq C_{1}, \\
& \max _{(x, t) \in \Omega}\left|\frac{\partial^{N+1} f(x, \zeta)}{\partial t^{N+1}}\right| \leq C_{2}, \\
& \max _{(x, t) \in \Omega}\left|\frac{\partial^{M+N+2} f\left(\xi^{\prime}, \zeta^{\prime}\right)}{\partial x^{M+1} \partial t^{N+1}}\right| \leq C_{3} .
\end{aligned}
$$

We estimate the factors $\left\|\prod_{i=0}^{M}\left(x-x_{i}\right)\right\|_{L^{\infty}}$ and $\left\|\prod_{k=0}^{N}\left(t-t_{k}\right)\right\|_{L^{\infty}}$.

Let $t=\frac{T}{2}(\tau+1)$. By the affine mapping between intervals $[0, T]$ and $[-1,1]$,

$$
\begin{aligned}
& \max _{t \in[0, T]}\left|\prod_{k=0}^{N}\left(t-t_{k}\right)\right|=\max _{\tau \in[-1,1]}\left|\prod_{k=0}^{N} \frac{T}{2}\left(\tau-\tau_{k}\right)\right|=\left(\frac{T}{2}\right)^{N+1} \max _{\tau \in[-1,1]}\left|\prod_{k=0}^{N}\left(\tau-\tau_{k}\right)\right| \\
= & \left(\frac{T}{2}\right)^{N+1} \max _{\tau \in[-1,1]}\left|\frac{\mathscr{P}_{N+1}^{(\alpha, \beta)}(\tau)}{\kappa_{N}^{(\alpha, \beta)}}\right|,
\end{aligned}
$$

where $\kappa_{N}^{(\alpha, \beta)}=\frac{\Gamma(2 N+\alpha+\beta+1)}{2^{N} N ! \Gamma(N+\alpha+\beta+1)}$ is the coefficient of $\mathscr{P}_{N+1}^{(\alpha, \beta)}(\tau)$ and $\tau_{k}=\frac{2}{T} t_{k}-1, t_{1}=0, t_{k}$, $(k=1, \cdots, N)$ are zeros of $\mathscr{P}_{T, N}^{(\alpha, \beta+1)}(2 t-T)$. By the same way, we have

$$
\max _{x \in[-1,1]}\left|\prod_{i=0}^{M}\left(x-x_{i}\right)\right|=\max _{x \in[-1,1]}\left|\frac{\mathscr{P}_{M+1}^{(\alpha, \beta)}(x)}{\kappa_{M}^{(\alpha, \beta)}}\right|,
$$

where $\kappa_{M}^{(\alpha, \beta)}=\frac{\Gamma(2 M+\alpha+\beta+1)}{2^{M} M ! \Gamma(M+\alpha+\beta+1)}$ is the coefficient of $\mathscr{P}_{M+1}^{(\alpha, \beta)}(x)$ and $x_{0}=-1, x_{M}=1, x_{i},(i=$ $1, \cdots, M-1)$ are the zeros of $\partial_{x} \mathscr{P}_{M}^{(\alpha, \beta)}(x)$.

By Lemma 5.1,

$$
\max _{-1 \leq x \leq 1}\left|\mathscr{P}_{M+1}^{(\alpha, \beta)}(x)\right| \leq K(M+1)^{q}, \quad \alpha, \beta>-1
$$

where $q=\max \left(\alpha, \beta,-\frac{1}{2}\right)$ and $K$ is a positive constant. On the interval $[-1,1]$, it reaches the maximum of $\left|\mathscr{P}_{M+1}^{(\alpha, \beta)}(x)\right|$ at $x=1$ and $\alpha \geq \beta, \alpha \geq-\frac{1}{2}$ [21],

$$
\max _{-1 \leq x \leq 1}\left|\mathscr{P}_{M+1}^{(\alpha, \beta)}(x)\right|=\mathscr{P}_{M+1}^{(\alpha, \beta)}(1)=\frac{\Gamma(M+\alpha+2)}{(M+1) ! \Gamma(\alpha+1)}=\mathcal{O}\left((M+1)^{\alpha}\right) .
$$


Applying (5.7)-(5.11), Eq. (5.5) reads:

$$
\begin{aligned}
M_{1}= & \left\|f(x, t)-f_{N, M}(x, t)\right\|_{L^{\infty}} \\
\leq & C_{1}^{\prime} \frac{(M+1)^{q}}{(M+1) ! \kappa_{M}^{(\alpha, \beta)}}+C_{2}^{\prime} \frac{(T / 2)^{N+1}(N+1)^{q}}{(N+1) ! \kappa_{N}^{(\alpha, \beta)}} \\
& \quad+C_{3}^{\prime} \frac{(M+1)^{q}(T / 2)^{N+1}(N+1)^{q}}{(M+1) !(N+1) ! \kappa_{M}^{(\alpha, \beta)} \kappa_{N}^{(\alpha, \beta)}} \\
= & C_{1}^{\prime} Q_{1}+C_{2}^{\prime} Q_{2}+C_{3}^{\prime} Q_{1} Q_{2} .
\end{aligned}
$$

By the same process, we have

$$
\begin{aligned}
M_{2}= & \left\|g(x, t)-g_{N, M}(x, t)\right\|_{L^{\infty}} \\
\leq & H_{1}^{\prime} \frac{(M+1)^{q}}{(M+1) ! \kappa_{M}^{(\alpha, \beta)}}+H_{2}^{\prime} \frac{(T / 2)^{N+1}(N+1)^{q}}{(N+1) ! \kappa_{N}^{(\alpha, \beta)}} \\
& \quad+H_{3}^{\prime} \frac{(M+1)^{q}(T / 2)^{N+1}(N+1)^{q}}{\kappa_{M}^{(M+1) !(N+1) !(\alpha, \beta)} \kappa_{N}^{(\alpha, \beta)}} \\
& =H_{1}^{\prime} Q_{1}+H_{2}^{\prime} Q_{2}+H_{3}^{\prime} Q_{1} Q_{2},
\end{aligned}
$$

where $H_{1}^{\prime}, H_{2}^{\prime}, H_{3}^{\prime}$ are constants and $q=\max \left(\alpha, \beta,-\frac{1}{2}\right)$. So

$$
\left\|u(x, t)-u_{N, M}(x, t)\right\|_{L^{\infty}} \leq\left(\left(C_{1}^{\prime} Q_{1}+C_{2}^{\prime} Q_{2}+C_{3}^{\prime} Q_{1} Q_{2}\right)^{2}+\left(H_{1}^{\prime} Q_{1}+H_{2}^{\prime} Q_{2}+H_{3}^{\prime} Q_{1} Q_{2}\right)^{2}\right)^{\frac{1}{2}},
$$

where

$$
Q_{1}=\frac{(M+1)^{q}}{(M+1) ! \kappa_{M}^{(\alpha, \beta)}}, \quad Q_{2}=\frac{(T / 2)^{N+1}(N+1)^{q}}{(N+1) ! \kappa_{N}^{(\alpha, \beta)}},
$$

The theorem is proved.

Then, we prove the above error estimate in $L^{2}$-norm.

Theorem 5.2. Let $u(x, t)=f(x, t)+i g(x, t)$ be the exact solution of the original problem (3.1), and $f(x, t), g(x, t)$ is sufficiently smooth. $u_{N, M}(x, t)=f_{N, M}(x, t)+i g_{N, M}(x, t)$ is approximate solution of the full-discrete problem (4.7), then the following error estimate holds:

$$
\left\|u(x, t)-u_{N, M}(x, t)\right\|_{L^{2}} \leq\left|\left(C_{1}^{*} Q_{1}^{*}+C_{2}^{*} Q_{2}^{*}+C_{3}^{*} Q_{1}^{*} Q_{2}^{*}\right)^{2}+\left(H_{1}^{*} Q_{1}+H_{2}^{*} Q_{2}+H_{3}^{*} Q_{1}^{*} Q_{2}^{*}\right)^{2}\right|^{\frac{1}{2}},
$$

where

$$
Q_{1}^{*}=\frac{(M+1)^{q}}{(M+1) ! \kappa_{M}^{(\alpha, \beta)}}, \quad Q_{2}^{*}=\frac{(T / 2)^{\frac{2 N-\alpha-\beta+1}{2}}(N+1)^{q}}{(N+1) ! \kappa_{N}^{(\alpha, \beta)}},
$$

and $C_{1}^{*}, C_{2}^{*}, C_{3}^{*}, H_{1}^{*}, H_{2}^{*}, H_{3}^{*}$ are constants, $q=\max \left(\alpha, \beta,-\frac{1}{2}\right)$ and $\kappa_{M}^{(\alpha, \beta)}$ is the coefficient of $\mathscr{P}_{M+1}^{(\alpha, \beta)}(x)$. 
Proof. Due to the best approximation in $L^{2}, \forall v_{N, M}(x, t) \in \mathcal{P}_{N, M}^{\alpha, \beta}$, we obtain the following inequality

$$
\left\|f(x, t)-f_{N, M}(x, t)\right\|_{L^{2}} \leq\left\|f(x, t)-v_{N, M}(x, t)\right\|_{L^{2}},
$$

where $v_{N, M}(x, t)$ denotes the interpolating polynomial for $f(x, t)$ at $\left(x_{i}, t_{k}\right)$, where $\left\{x_{i}\right\}$ is the JGL interpolation of $\mathscr{P}_{M}^{(\alpha, \beta)}(x)$ and $\left\{t_{k}\right\}$ is the JGR interpolation of $\mathscr{P}_{T, K}^{(\alpha, \beta)}(t)$.

$$
\begin{array}{r}
f(x, t)-v_{N, M}(x, t)=\frac{\partial^{M+1} f(\xi, t)}{(M+1) ! \partial x^{M+1}} \prod_{i=0}^{M}\left(x-x_{i}\right)+\frac{\partial^{N+1} f(x, \zeta)}{(N+1) ! \partial t^{N+1}} \prod_{i=0}^{N}\left(t-t_{k}\right) \\
-\frac{\partial^{M+N+2} f\left(\xi^{\prime}, \zeta^{\prime}\right)}{(M+1) !(N+1) ! \partial x^{M+1} \partial t^{N+1}} \prod_{i=0}^{M}\left(x-x_{i}\right) \prod_{i=0}^{N}\left(t-t_{k}\right),
\end{array}
$$

where $\xi, \xi^{\prime} \in[-1,1], \zeta, \zeta^{\prime} \in[0, T]$,

$$
\begin{aligned}
& \left\|f(x, t)-v_{N, M}(x, t)\right\|_{L^{2}} \\
\leq & \left\|\frac{\partial^{M+1} f(\xi, t)}{\partial x^{M+1}}\right\|_{L^{2}} \frac{\left\|\prod_{i=0}^{M}\left(x-x_{i}\right)\right\|_{L^{2}}}{(M+1) !}+\left\|\frac{\partial^{N+1} f(x, \zeta)}{\partial t^{N+1}}\right\|_{L^{2}} \frac{\left\|\prod_{i=0}^{N}\left(t-t_{k}\right)\right\|_{L^{2}}}{(N+1) !} \\
& +\left\|\frac{\partial^{M+N+2} f\left(\xi^{\prime}, \zeta^{\prime}\right)}{\partial x^{M+1} \partial t^{N+1}}\right\|_{L^{2}} \frac{\left\|\prod_{i=0}^{M}\left(x-x_{i}\right)\right\|_{L^{2}}\left\|\prod_{i=0}^{N}\left(t-t_{k}\right)\right\|_{L^{2}}}{(M+1) !(N+1) !} .
\end{aligned}
$$

There exist constants $C_{1}, C_{2}$ and $C_{3}$ such that

$$
\begin{aligned}
& \left\|\frac{\partial^{M+1} f(\xi, t)}{\partial x^{M+1}}\right\|_{L^{2}}=\left[\int_{0}^{T}\left(\frac{\partial^{M+1} f(\xi, t)}{\partial x^{M+1}}\right)^{2} \omega^{(\alpha, \beta)}(t) d t\right]^{\frac{1}{2}} \leq C_{1} \\
& \left\|\frac{\partial^{N+1} f(x, \zeta)}{\partial t^{N+1}}\right\|_{L^{2}}=\left[\int_{-1}^{1}\left(\frac{\partial^{N+1} f(x, \zeta)}{\partial t^{N+1}}\right)^{2} \omega^{(\alpha, \beta)}(x) d x\right]^{\frac{1}{2}} \leq C_{2} \\
& \left\|\frac{\partial^{M+N+2} f\left(\zeta^{\prime}, \zeta^{\prime}\right)}{\partial x^{M+1} \partial t^{N+1}}\right\|_{L^{2}} \leq C_{3} .
\end{aligned}
$$

Then

$$
\begin{aligned}
& \left\|\prod_{i=0}^{M}\left(x-x_{i}\right)\right\|_{L^{2}} \\
= & {\left[\int_{-1}^{1}\left(\prod_{i=0}^{M}\left(x-x_{i}\right)\right)^{2} \omega^{(\alpha, \beta)}(x) d x\right]^{\frac{1}{2}}=\left[\int_{-1}^{1}\left(\frac{\mathscr{P}_{M+1}^{(\alpha, \beta)}(x)}{\kappa_{M}^{(\alpha, \beta)}}\right)^{2} \omega^{(\alpha, \beta)}(x) d x\right]^{\frac{1}{2}} } \\
\leq & \frac{\sqrt{2}}{\kappa_{M}^{(\alpha, \beta)}}\left[\max _{x \in[-1,1]}\left|\left(\mathscr{P}_{M+1}^{(\alpha, \beta)}(x)\right)^{2} \omega^{(\alpha, \beta)}(x)\right|\right]^{\frac{1}{2}} \leq \frac{K_{1}}{\kappa_{M}^{(\alpha, \beta)}} \max _{x \in[-1,1]}\left|\mathscr{P}_{M+1}^{(\alpha, \beta)}(x)\right|,
\end{aligned}
$$

where $K_{1}$ is constant, $\kappa_{M}^{(\alpha, \beta)}=\frac{\Gamma(2 M+\alpha+\beta+1)}{2^{M} M ! \Gamma(M+\alpha+\beta+1)}$ is the coefficient of $\mathscr{P}_{M+1}^{(\alpha, \beta)}(x), \omega^{(\alpha, \beta)}(x)=$ $(1-x)^{\alpha}(1+x)^{\beta}$ is weight function and $x_{0}=-1, x_{M}=1, x_{i},(i=1, \cdots, M-1)$ are the zeros of $\partial_{x} \mathscr{P}_{M}^{(\alpha, \beta)}(x)$. 
Let $t=\frac{T}{2}(\tau+1)$, then $\tau \in[-1,1]$. With the weight function $\omega_{T}^{(\alpha, \beta)}(t)=(T-t)^{\alpha} t^{\beta}$ over $I=[0, T]$, we have

$$
\begin{aligned}
\omega_{T}^{(\alpha, \beta)}(t) & =(T-t)^{\alpha} t^{\beta}=\left(\frac{2}{T}\right)^{\alpha+\beta}\left(\frac{T}{2}\right)^{\alpha+\beta}\left(L-\frac{T}{2}(\tau+1)\right)^{\alpha}\left(\frac{T}{2}(\tau+1)\right)^{\beta} \\
& =\left(\frac{2}{T}\right)^{\alpha+\beta}(1-\tau)^{\alpha}(1+\tau)^{\beta}=\left(\frac{2}{T}\right)^{\alpha+\beta} \omega^{(\alpha, \beta)}(\tau), \quad \tau \in[-1,1] .
\end{aligned}
$$

Next,

$$
\begin{aligned}
\left\|\prod_{k=0}^{N}\left(t-t_{k}\right)\right\|_{L^{2}} & =\left[\int_{0}^{T}\left(\prod_{k=0}^{N}\left(t-t_{k}\right)\right)^{2} \omega_{T}^{(\alpha, \beta)}(t) d t\right]^{\frac{1}{2}} \\
& =\left[\int_{-1}^{1}\left(\left(\frac{T}{2}\right)^{N} \prod_{k=0}^{N}\left(\tau-\tau_{k}\right)\right)^{2}\left(\frac{2}{T}\right)^{\alpha+\beta-1} \omega^{(\alpha, \beta)}(\tau) d \tau\right]^{\frac{1}{2}} \\
& =\left(\frac{T}{2}\right)^{\frac{2 N-\alpha-\beta+1}{2}}\left[\int_{-1}^{1}\left(\prod_{k=0}^{N}\left(\tau-\tau_{k}\right)\right)^{2} \omega^{(\alpha, \beta)}(\tau)\right]^{\frac{1}{2}} \\
& =\left(\frac{T}{2}\right)^{\frac{2 N-\alpha-\beta+1}{2}}\left[\int_{-1}^{1}\left(\frac{\mathscr{P}_{N+1}^{(\alpha, \beta)}(\tau)}{\kappa_{N}^{(\alpha, \beta)}}\right)^{2} \omega^{(\alpha, \beta)}(\tau) d \tau\right]^{\frac{1}{2}} \\
& \leq\left(\frac{T}{2}\right)^{\frac{2 N-\alpha-\beta+1}{2}} \frac{\sqrt{2}}{\kappa_{N}^{(\alpha, \beta)}}\left[\max _{\tau \in[-1,1]} \mid\left(\mathscr{P}_{N+1}^{(\alpha, \beta)}(\tau)\right)^{2} \omega^{(\alpha, \beta)}(\tau)\right]^{\frac{1}{2}} \\
& \leq\left(\frac{T}{2}\right)^{\frac{2 N-\alpha-\beta+1}{2}} \frac{K_{2}}{\kappa_{N}^{(\alpha, \beta)}} \max _{\tau \in[-1,1]}\left|\mathscr{P}_{N+1}^{(\alpha, \beta)}(\tau)\right|,
\end{aligned}
$$

where $K_{2}$ is constant, $\kappa_{N}^{(\alpha, \beta)}=\frac{\Gamma(2 N+\alpha+\beta+1)}{2^{N} N ! \Gamma(N+\alpha+\beta+1)}$ is the leading coefficient of $\mathscr{P}_{N+1}^{(\alpha, \beta)}(\tau)$ and $\tau_{k}=\frac{2}{T} t_{k}-1, t_{1}=0, t_{k},(k=1, \cdots, N)$ are zeros of $\mathscr{P}_{T, N}^{(\alpha, \beta+1)}(2 t-T)$.

By Lemma 5.1,

$$
\max _{-1 \leq x \leq 1}\left|\mathscr{P}_{M+1}^{(\alpha, \beta)}(x)\right| \leq K(M+1)^{q}, \quad \alpha, \beta>-1,
$$

where $q=\max \left(\alpha, \beta,-\frac{1}{2}\right)$ and $K$ is a positive. Then

$$
\max _{-1 \leq x \leq 1}\left|\mathscr{P}_{M+1}^{(\alpha, \beta)}(x)\right|=\mathscr{P}_{M+1}^{(\alpha, \beta)}(1)=\frac{\Gamma(M+\alpha+2)}{(M+1) ! \Gamma(\alpha+1)}=\mathcal{O}\left((M+1)^{\alpha}\right) .
$$

Applying Eqs. (5.14) and (5.19), Eq. (5.12) reads

$$
\begin{aligned}
M_{1} & =\left\|f(x, t)-f_{N, M}(x, t)\right\|_{L^{2}} \\
& \leq C_{1}^{*} \frac{(M+1)^{q}}{\kappa_{M}^{(\alpha, \beta)}(M+1) !}+C_{2}^{*} \frac{(T / 2)^{\frac{2 N-\alpha-\beta+1}{2}}(N+1)^{q}}{\kappa_{N}^{(\alpha, \beta)}(N+1) !}
\end{aligned}
$$




$$
\begin{gathered}
+C_{3}^{*} \frac{(M+1)^{q}(T / 2)^{\frac{2 N-\alpha-\beta+1}{2}}(N+1)^{q}}{\kappa_{M}^{(\alpha, \beta)} \kappa_{N}^{(\alpha, \beta)}(M+1) !(N+1) !} \\
=C_{1}^{*} Q_{1}^{*}+C_{2}^{*} Q_{2}^{*}+C_{3}^{*} Q_{1}^{*} Q_{2}^{*} .
\end{gathered}
$$

By the same process, we have

$$
\begin{aligned}
M_{2}= & \left\|g(x, t)-g_{N, M}(x, t)\right\|_{L^{2}} \\
\leq & H_{1}^{*} \frac{(M+1)^{q}}{(M+1) ! \kappa_{M}^{(\alpha, \beta)}}+H_{2}^{*} \frac{(T / 2)^{\frac{2 N-\alpha-\beta+1}{2}}(N+1)^{q}}{(N+1) ! \kappa_{N}^{(\alpha, \beta)}} \\
& \quad+H_{3}^{*} \frac{(M+1)^{q}(T / 2)^{\frac{2 N-\alpha-\beta+1}{2}}(N+1)^{q}}{(M+1) !(N+1) ! \kappa_{M}^{(\alpha, \beta)} \kappa_{N}^{(\alpha, \beta)}} \\
= & H_{1}^{*} Q_{1}^{*}+H_{2}^{*} Q_{2}^{*}+H_{3}^{*} Q_{1}^{*} Q_{2}^{*},
\end{aligned}
$$

where $H_{1}^{\prime}, H_{2}^{\prime}, H_{3}^{\prime}$ are constants and $q=\max \left(\alpha, \beta,-\frac{l}{2}\right)$. Finally,

$$
\left\|u(x, t)-u_{N, M}(x, t)\right\|_{L^{2}} \leq \sqrt{M_{1}^{2}+M_{2}^{2}} .
$$

The theorem is proved.

\section{Numerical tests}

In this section, we give numerical examples to illustrate the high order accuracy and efficiency of our methods.

Example 6.1 (A linear equation). The domain is $(x, t) \in(-1,1) \times(0,1)$. The $a=3 / 2$ fractional order Ginzburg-Landau equation is solved:

$$
\begin{aligned}
& u_{t}+(4+i)(-\Delta)^{3 / 4} u-u=f_{1}(x, t), \\
& u(x, 0)=0 \\
& u(-1, t)=u(1, t)=0,
\end{aligned}
$$

where $f_{1}$ is defined such that the exact solution is

$$
u(x, t)=\left(e^{4 t^{2}}-1\right)\left((x+1)^{3}(1-x)^{2}+i(x+1)^{2}(1-x)^{3}\right) .
$$

In the space direction $x$, we use $\mathscr{P}_{M+2}$ Jacobi-Gauss-Lobatto orthogonal polynomials on $[-1,1]$, i.e., $x_{0}=-1$ and $x_{M+1}=1$, with the weight $(x+1)^{1-a}(1-x)^{1-a}, a=3 / 2$. In the time direction $t$, we use $\mathscr{P}_{N}$ left Jacobi-Gauss-Radau orthogonal polynomials on $[0,1]$, i.e., $t_{0}=0$. The number of collocation points is $M N$, instead of $(M+2)(N+1)$, as the initial values and boundary values are given. The numerical solution and the exact solution at 


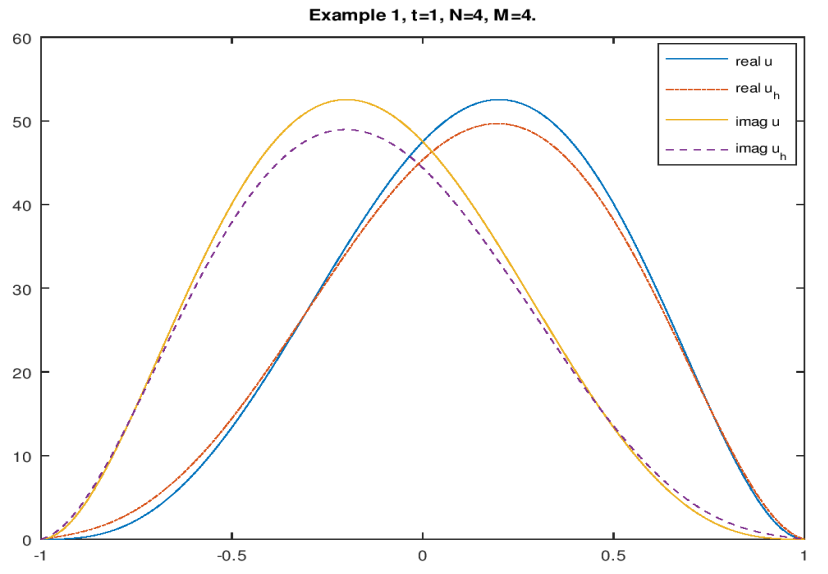

Figure 1: The numerical solution and exact solution of (6.2), $a=3 / 2$.

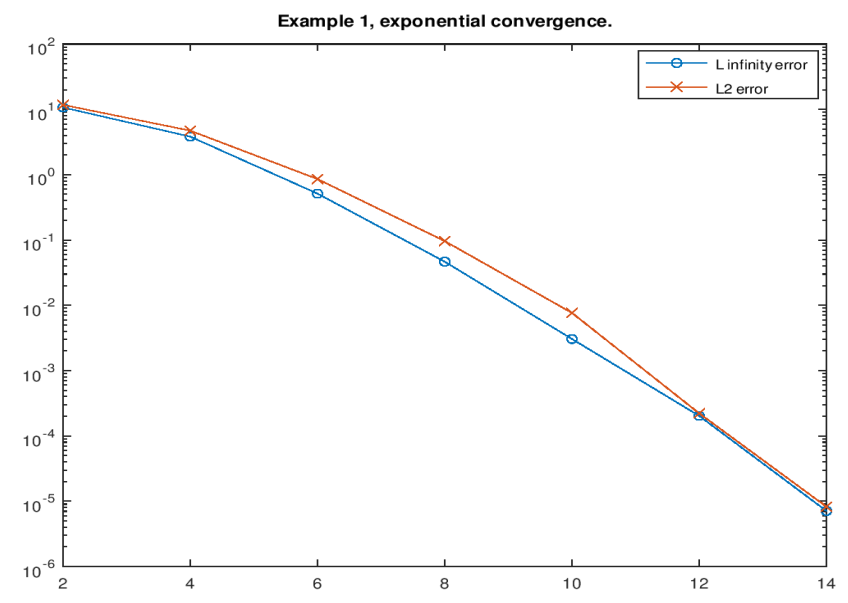

Figure 2: The (exponential) convergence of the numerical solution for (6.2), $a=3 / 2$.

Table 1: The $L^{\infty}$ error and the $L^{2}$ error at $t=1$ for (6.1).

\begin{tabular}{||c|c|c||}
\hline$M, N$ & $\left\|u-u_{h}\right\|_{L^{\infty}}$ & $\left\|u-u_{h}\right\|_{L^{2}}$ \\
\hline 2,2 & $1.0708 \mathrm{E}+001$ & $1.1732 \mathrm{E}+001$ \\
4,4 & $3.8265 \mathrm{E}-000$ & $4.7214 \mathrm{E}-000$ \\
6,6 & $5.1255 \mathrm{E}-001$ & $8.5651 \mathrm{E}-001$ \\
8,8 & $4.6525 \mathrm{E}-002$ & $9.6116 \mathrm{E}-002$ \\
10,10 & $3.0494 \mathrm{E}-003$ & $7.6455 \mathrm{E}-003$ \\
12,12 & $2.0248 \mathrm{E}-004$ & $2.2147 \mathrm{E}-004$ \\
14,14 & $7.0797 \mathrm{E}-006$ & $8.2330 \mathrm{E}-006$ \\
\hline
\end{tabular}


time $t=1$ are plotted in Fig. 1 , for $M=4$ and $N=4$. We list the numerical $L^{\infty}$ error and the $L^{2}$ error at time $t=1$ in Table 1 . In addition we plot the error in the semi-logy scale in Fig. 2 to show the exponential convergence. Moreover, $u$ and $u_{h}$ are the exact solution and approximate solution in table and figure, respectively.

Example 6.2 (A nonlinear equation). The fractional order Ginzburg-Landau equation to be solved is

$$
\begin{aligned}
& u_{t}+(6-i)(-\Delta)^{3 / 4} u+\left(2-i \frac{1}{2}\right)|u|^{2} u-u=f_{2}(x, t), \quad(x, t) \in(-1,1) \times(0,1), \\
& u(x, 0)=0, \\
& u(-1, t)=u(1, t)=0,
\end{aligned}
$$

where $f_{2}$ is chosen so that the exact solution is

$$
u(x, t)=\sin t^{2}(x+1) x(1-x)(1-i) .
$$

Again, we use the tensor product basis of $\left(-\frac{1}{2},-\frac{1}{2}\right)$ Jacobi-Gauss-Lobatto $\mathscr{P}_{M+2}(x)$ polynomials and left Jacobi-Gauss-Radau $\mathscr{P}_{N}(t)$ polynomials. The numerical solution and the exact solution at time $t=1$ are plotted in Fig. 3, for $M=2$ and $N=2$. We list the numerical $L^{\infty}$ error and the $L^{2}$ error at time $t=1$ in Table 2 . We plot the error in the semilogy scale in Fig. 4 to see its exponential convergence. Moreover, $u$ and $u_{h}$ are the exact solution and approximate solution in table and figure, respectively.

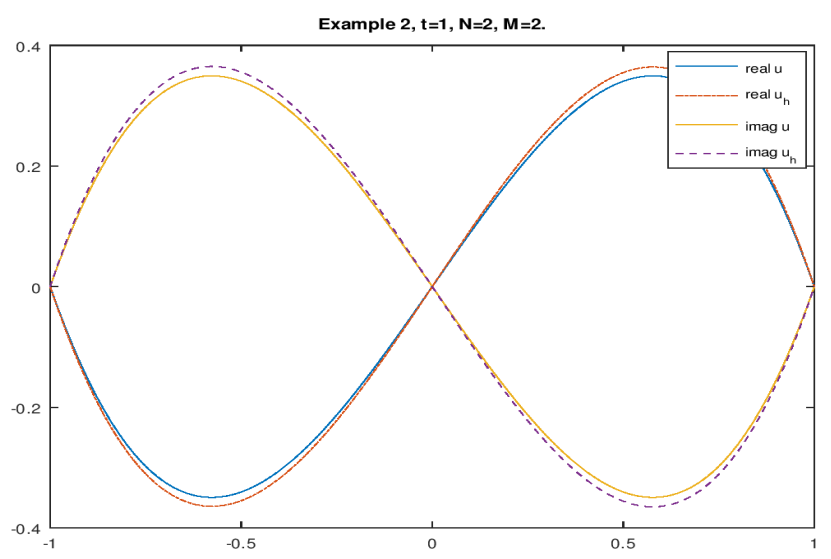

Figure 3: The numerical solution and exact solution of (6.4), $a=3 / 2$.

Example 6.3 (A 2D nonlinear equation). The domain is $(x, y, t) \in(-1,1) \times(-1,1) \times(0,1)$. A 2D $a=3 / 2$ fractional order Ginzburg-Landau equation is solved:

$$
\begin{aligned}
& u_{t}+a_{1}\left(-\frac{\partial^{3 / 2} u}{\partial|x|^{3 / 2}}-\frac{\partial^{3 / 2} u}{\partial|y|^{3 / 2}}\right)+a_{2}|u|^{2} u-a_{3} u=f_{3}, \\
& u(x, y, 0)=0, \quad u( \pm 1, \pm 1, t)=0
\end{aligned}
$$




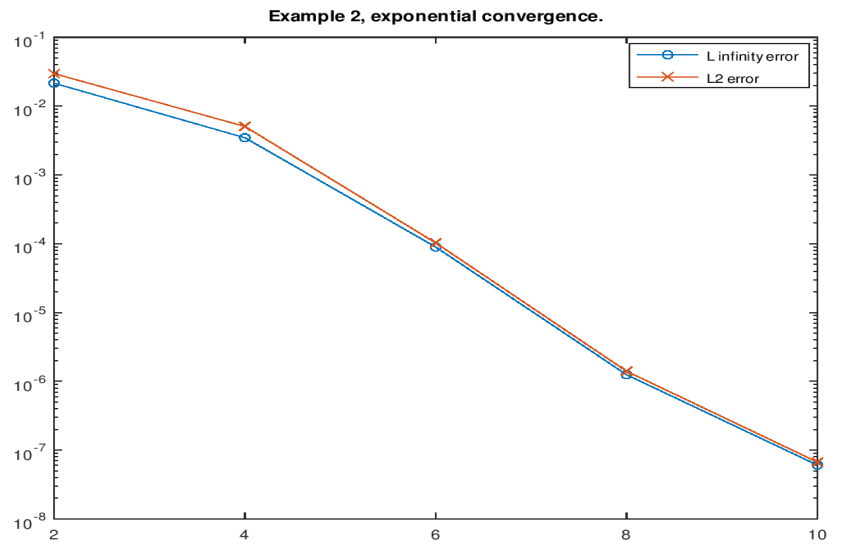

Figure 4: The (exponential) convergence of the numerical solution for (6.4).

Table 2: The $L^{\infty}$ error and the $L^{2}$ error at $t=1$, for (6.3).

\begin{tabular}{||c|c|c||}
$M, N$ & $\left\|u-u_{h}\right\|_{L^{\infty}}$ & $\left\|u-u_{h}\right\|_{L^{2}}$ \\
\hline 2,2 & $2.1544 \mathrm{E}-002$ & $2.9686 \mathrm{E}-002$ \\
4,4 & $3.4703 \mathrm{E}-003$ & $5.0677 \mathrm{E}-003$ \\
6,6 & $8.9564 \mathrm{E}-005$ & $1.0299 \mathrm{E}-004$ \\
8,8 & $1.2479 \mathrm{E}-006$ & $1.4046 \mathrm{E}-006$ \\
10,10 & $6.0722 \mathrm{E}-008$ & $6.7026 \mathrm{E}-008$ \\
\hline
\end{tabular}

where

$$
a_{1}=6-i, \quad a_{2}=2-i, \quad a_{3}=8,
$$

and

$$
\begin{aligned}
f_{3}=\left(2 t \mathrm{e}^{t}\right. & \left.+t^{2} \mathrm{e}^{t}\right)\left(\left(x-x^{5}\right)\left(y-y^{3}\right)-i\left(x-x^{5}\right)\left(y-y^{3}\right)\right) \\
& -\frac{a_{1}}{2}\left(\frac{(4-4 i) y(y+1)(y-1)}{\sqrt{x+1} \sqrt{\pi}}-\frac{(2-2 i)(-x+i)(x+1)(x+i)(x-1) x}{\sqrt{y+1} \sqrt{\pi}}\right. \\
& +\frac{\left(\frac{8}{7}-\frac{8 i}{7}\right) \sqrt{x+1}\left(16 x^{3}-8 x^{2}+6 x-5\right) y(y+1)(y-1)}{\sqrt{\pi}} \\
& -\frac{(4-4 i) \sqrt{y+1}(-x+i)(x+1)(x+i)(x-1) x(2 y-1)}{\sqrt{\pi}} \\
& -\frac{(4-4 i) y(y+1)(y-1)}{\sqrt{1-x} \sqrt{\pi}}+\frac{(2-2 i)(-x+i)(x+1)(x+i)(x-1) x}{\sqrt{1-y} \sqrt{\pi}} \\
& +\frac{\left(\frac{8}{7}-\frac{8 i}{7}\right) \sqrt{1-x}\left(16 x^{3}+8 x^{2}+6 x+5\right) y(y+1)(y-1)}{\sqrt{\pi}}
\end{aligned}
$$




$$
\begin{aligned}
& \left.-\frac{(4-4 i) \sqrt{1-y}(-x+i)(x+1)(x+i)(x-1) x(2 y+1)}{\sqrt{\pi}}\right) \sqrt{2} t^{2} \mathrm{e}^{t} \\
& -a_{3}\left(\left(x-x^{5}\right)\left(y-y^{3}\right)-i\left(x-x^{5}\right)\left(y-y^{3}\right)\right) t^{2} \mathrm{e}^{t}+2 a_{2}\left(x-x^{5}\right)^{2} \\
& \times\left(y-y^{3}\right)^{2}\left(\left(x-x^{5}\right)\left(y-y^{3}\right)-i\left(x-x^{5}\right)\left(y-y^{3}\right)\right) t^{6}\left(\mathrm{e}^{t}\right)^{3} .
\end{aligned}
$$

So the exact solution is

$$
u(x, y, t)=t^{2} e^{t}\left(x-x^{5}\right)\left(y-y^{3}\right)(1-i) .
$$

In the space directions, $x$ and $y$, we use $\mathscr{P}_{M+2}$ Jacobi-Gauss-Lobatto orthogonal polynomials on $[-1,1]$, i.e., $x_{0}=-1$ and $x_{M+1}=1$, with the weight $(x+1)^{1-a}(1-x)^{1-a}$ (and $(y+1)^{1-a}(1-y)^{1-a}$ in $y$-diretion), $a=3 / 2$. In computing the fractional derivative in $x$ direction, we use the left and right $\mathscr{P}_{M+2}$ Jacobi-Gauss-Radau quadrature points. In the time direction $t$, we use $\mathscr{P}_{N+1}$ left Jacobi-Gauss-Radau orthogonal polynomials on $[0,1]$, i.e., $t_{0}=0$. When computing the time-direction integrals, we use the $\mathscr{P}_{N+1}$ Jacobi-GaussRadau points. The number of collocation points is $M^{2} N$, instead of $(M+2)^{2}(N+1)$, as the initial values and boundary values are given.The numerical solution and the error at time $t=1$ are plotted in Fig. 5, for $M=4$ and $N=4$. We list the numerical $L^{\infty}$ error and
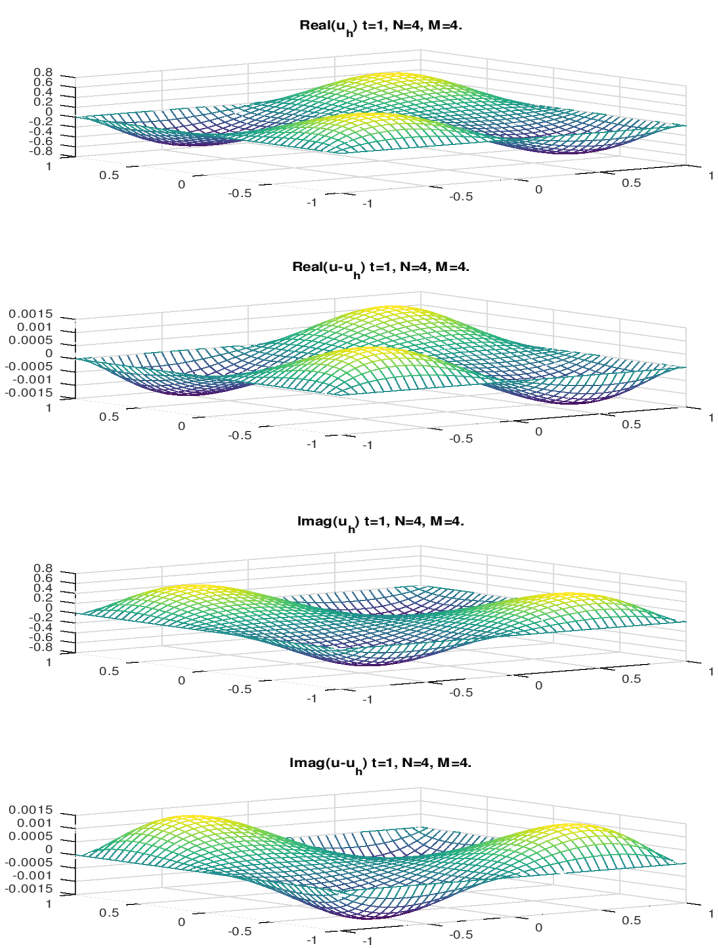

Figure 5: The numerical solution and the error for (6.7). 


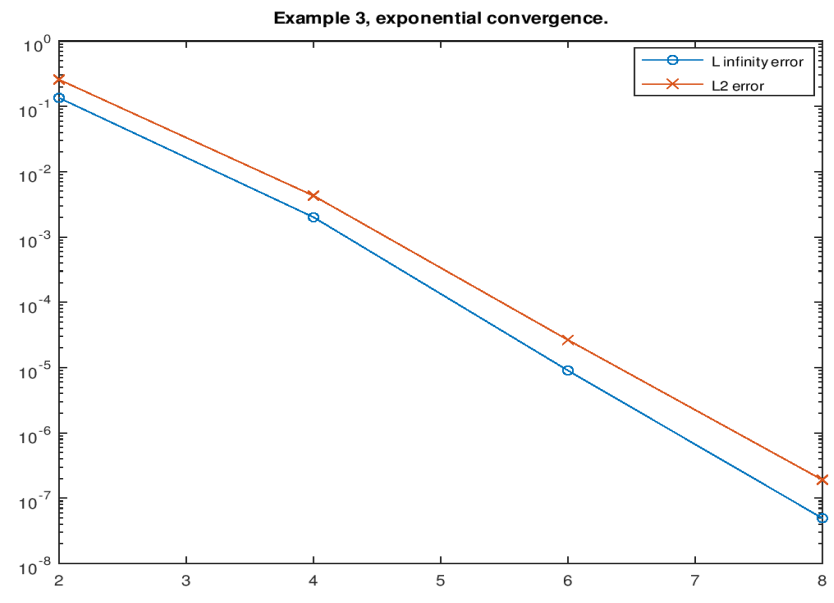

Figure 6: The (exponential) convergence of the numerical solution for (6.7).

Table 3: The $L^{\infty}$ error and the $L^{2}$ error at $t=1$, for 6.7 .

\begin{tabular}{||c|c|c||}
\hline$M, N$ & $\left\|u-u_{h}\right\|_{L^{\infty}}$ & $\left\|u-u_{h}\right\|_{L^{2}}$ \\
\hline 2,2 & $1.3481 \mathrm{E}-001$ & $2.5712 \mathrm{E}-001$ \\
4,4 & $2.0071 \mathrm{E}-003$ & $4.2842 \mathrm{E}-003$ \\
6,6 & $9.0217 \mathrm{E}-006$ & $2.6478 \mathrm{E}-005$ \\
8,8 & $4.9333 \mathrm{E}-008$ & $1.9056 \mathrm{E}-007$ \\
10,10 & $6.4998 \mathrm{E}-008$ & $3.0594 \mathrm{E}-007$ \\
\hline
\end{tabular}

the $L^{2}$ error at time $t=1$ in Table 3 . When $N>8$, the round-off error limits the converges of the method. We plot the error in the semi-logy scale in Fig. 6 to show the exponential convergence. Moreover, $u$ and $u_{h}$ are the exact solution and approximate solution in table and figure, respectively.

According to the results of above numerical tests and convergence analysis, we know that when the solution is sufficiently smooth, the method we proposed can achieve high precision with fewer indicators (taking fewer nodes in the interval) and it converges to the exact solution with the rate of exponential power. The convergence order of the method in [27] is two orders. In addition, the method we proposed is easier to be implemented for nonlinear problems and it is suitable for long-time calculations and for large $N$.

\section{Conclusions}

In this paper, we proposed an efficient and high precision algorithm based on JacobiGauss-Lobatto and Jacobi-Gauss-Radau collocation method. A collocation method is 
developed and applied in two successive steps. In the first part, the JGLC method is employed for space discretization. Then we converted the equation with its initial conditions into a system of ODEs with the time variable. In the second part, we solve the system of ODEs in time from step one based on the JGRC method. Then, we obtain an algebraic system and solve it by Newton's method. The proposed scheme has many advantages. First, it is easier to be implemented for nonlinear problems. Then, it is suitable for long-time calculations and for large $N$ (very high accuracy). In addition, we give the theoretical proof of the convergence of collocation method in both $L^{\infty}$-norm and $L^{2}$-norm. Finally, we give specific numerical examples. The numerical results confirm the validity of scheme for solving the fractional equations and the correctness of the conclusions. This indicates that the JGLC-JGRC method has a good prospect in solving space-fractional differential equations.

\section{Acknowledgements}

The author would like to thank the referees for the helpful suggestions. The work was supported by National Natural Science Foundation of China Project (Nos. 11671342, $11771369,11931003)$, Project of Scientific Research Fund of Hunan Provincial Science and Technology Department (Nos. 2018JJ2374, 2018WK4006, 2019YZ3003), Key Project of Hunan Provincial Department of Education (No. 17A210) and mega-grant of the Russian Federation Government (N 14.Y26.31.0013).

\section{References}

[1] I. S. ARANSON AND L. KRAmeR, The world of the complex Ginzburg-Landau equation, Rev. Mode. Phys, 74 (2002), pp. 99-143.

[2] V. E. TARASOV AND G. M. ZASLAVSKY, Fractional Ginzburg-Landau equation for fractal media, Phys. A, 354 (2005), pp. 249-261.

[3] Y. YANG, Y. Q. HUANG AND Y. ZHOU, Numerical solutions for solving time fractional FokkerPlanck equations based on spectral collocation methods, J. Comput. Appl. Math., 339 (2018), pp. 389-404.

[4] A. Milovanov And J. Rasmussen, Fractional generalization of the Ginzburg-Landau equation, an unconventional approach to critical phenomena in complex media, Phys. Lett. A, 337 (2005), pp. 75-80.

[5] A. Mvogo, A. Tambue, G. Ben-Bolie and T. Kofane, Localized numerical impulses solutions in diffuse neural networks modeled by the complex fractional Ginzburg-Landau equation, Commun. Nonlin. Sci. Numer. Simu., 39 (2016), pp. 396-410.

[6] Q. YANG, F. LIU AND I. TURNER, Numerical methods for fractional partial differential equations with Riesz space fractional derivatives, Appl. Math. Model, 34 (2010), pp. 200-218.

[7] P. ZHUANG, F. LIU, V. ANH AND I. TURNER, Numerical method for the variable-order fractional advection-diffusion equation with a nonlinear source term, J. Numer. Anal., 47 (2009), pp. 1760-1781. 
[8] Y. YANG, Y. Q. CHEN AND Y. Q. HUANG, Spectral-collocation method for fractional Fredholm integro-differential equations, J. Korean. Math. Soc., 51(1) (2014), pp. 203-224.

[9] Y. YANG, Y. P. CHEN, Y. Q. HuANG AND H. Y. WEI, Spectral collocation method for the time-fractional diffusion-wave equation and convergence analysis, J. Comput. Appl. Math., 73(6) (2017), pp. 1218-1232.

[10] V. MilLOT AND Y. SIER, On a fractional Ginzburg-Landau equation and 1/2-harmonic maps into spheres, Arch. Ration. Mech. Anal., 215 (2015), pp. 125-210.

[11] B. GUO AND Z. HUO, Well-posedness for the nonlinear fractional Schrödinger equation and inviscid limit behavior of solution for the fractional Ginzburg-Landau equation, Fract. Calc. Appl. Anal., 16 (2013), pp. 226-242.

[12] H. LU, S. Lu AND Z. FENG, Asymptotic dynamics of 2D fractional complex Ginzburg-Landau equation, Int. J. Bifurc. Chaos, 23 (2013), 1350202.

[13] V. TARAsov, Psi-series solution of fractional Ginzburg-Landau equation, J. Phys. A Math. Gen., 39 (2006), 8395.

[14] X. Pu AND B. GuO, Well-posedness and dynamics for the fractional Ginzburg-Landau equation, Appl. Anal., 92 (2013), pp. 318-334.

[15] Y. YANG, Y. P. CHEN AND Y. Q. HUANG, Spectral-collocation method for fractional Fredholm integro-differential equations, J. Korean. Math. Soc., 51(1) (2014), pp. 203-224.

[16] Y. YANG, Y. P. CHEN AND Y. Q. HUANG, Convergence analysis of the Jacobi spectral collocation method for fractional integro-differential equations, Acta. Math. Sci., 34(3) (2014), pp. 673-690.

[17] J. SHEN, T. TANG AND L. L. WANG, Spectral Methods: Algorithms, Analysis and Application, (2011).

[18] C. CANuto, M. Y. Hussaini, A. Quarteroni And T. A. ZANG, Spectral Methods: Fundamentals in Single Domains, Springer-Verlag, New York, 2006.

[19] M. GASCA AND T. SAUER, On the history of multivariate polynomial interpolation, J. Comput. Appl. Math., 122 (2000), pp. 23-35.

[20] M. MAIN AND L. M. DELVES, The convergence rates of expansions in Jacobi polynomials, Numer. Math., 27 (1977), pp. 219-225.

[21] H. BAVINCK, On absolute convergence of Jacobi series, J. Appr. Theory, 4 (1971), pp. 387-400.

[22] X. MA AND C. HUANG, Spectral collocation method for linear fractional integro-differential equations, Appl. Math. Model, 38 (2014), pp. 1434-1448.

[23] M. R. EslahChi, M. Dehghan And M. Parvizi, Application of the collocation method for solving nonlinear fractional integro-differential equations, J. Comput. Appl. Math., 257 (2014), pp. 105-128.

[24] A. H. BHRAWY AND M. A. ZAKY, Highly accurate numerical schemes for multi-dimensional space variable-order fractional Schrödinger equations, Appl. Math., 73 (2017), pp. 1100-1117.

[25] T. WANG AND B. GUO, Analysis of some finite difference schemes for two-dimensional GinzburgLandau equation, Numer. Methods Partial Differenial Equations, 27 (2011), pp. 1340-1363.

[26] Y. YANG, Y. Q. HUANG AND Y. ZHOU, Numerical simulation of time fractional Cable equations and convergence analysis, Methods Partial Differenial Equations, 34 (2018), pp. 1556-1576.

[27] M. LI, C. M. HUANG AND N. WANG, Galerkin finite element method for the nonlinear fractional Ginzburg-Landau equation, Appl. Numer. Math., 118 (2017), pp. 131-149.

[28] Y. YANG, W. Y. QIAO, J. D. WANG AND S. Y. ZHANG, Spectral collocation methods for nonlinear coupled time fractional Nernest-Planck equations in two dimensions and its convergence analysis, Comput. Math. Appl., https://doi.org/10.1016/j. camwa.2018.12.018. 